Soluble adenylyl cyclase-mediated cAMP signaling and the putative role of PKA and EPAC in cerebral mitochondrial function

Jakobsen, Emil; Lange, Sofie C; Bak, Lasse K

Published in:

Journal of Neuroscience Research

DOI:

10.1002/jnr.24477

Publication date:

2019

Document version

Publisher's PDF, also known as Version of record

Document license:

CC BY

Citation for published version (APA):

Jakobsen, E., Lange, S. C., \& Bak, L. K. (2019). Soluble adenylyl cyclase-mediated cAMP signaling and the putative role of PKA and EPAC in cerebral mitochondrial function. Journal of Neuroscience Research, 97(8), 1018-1038. https://doi.org/10.1002/jnr.24477 


\section{gibco}

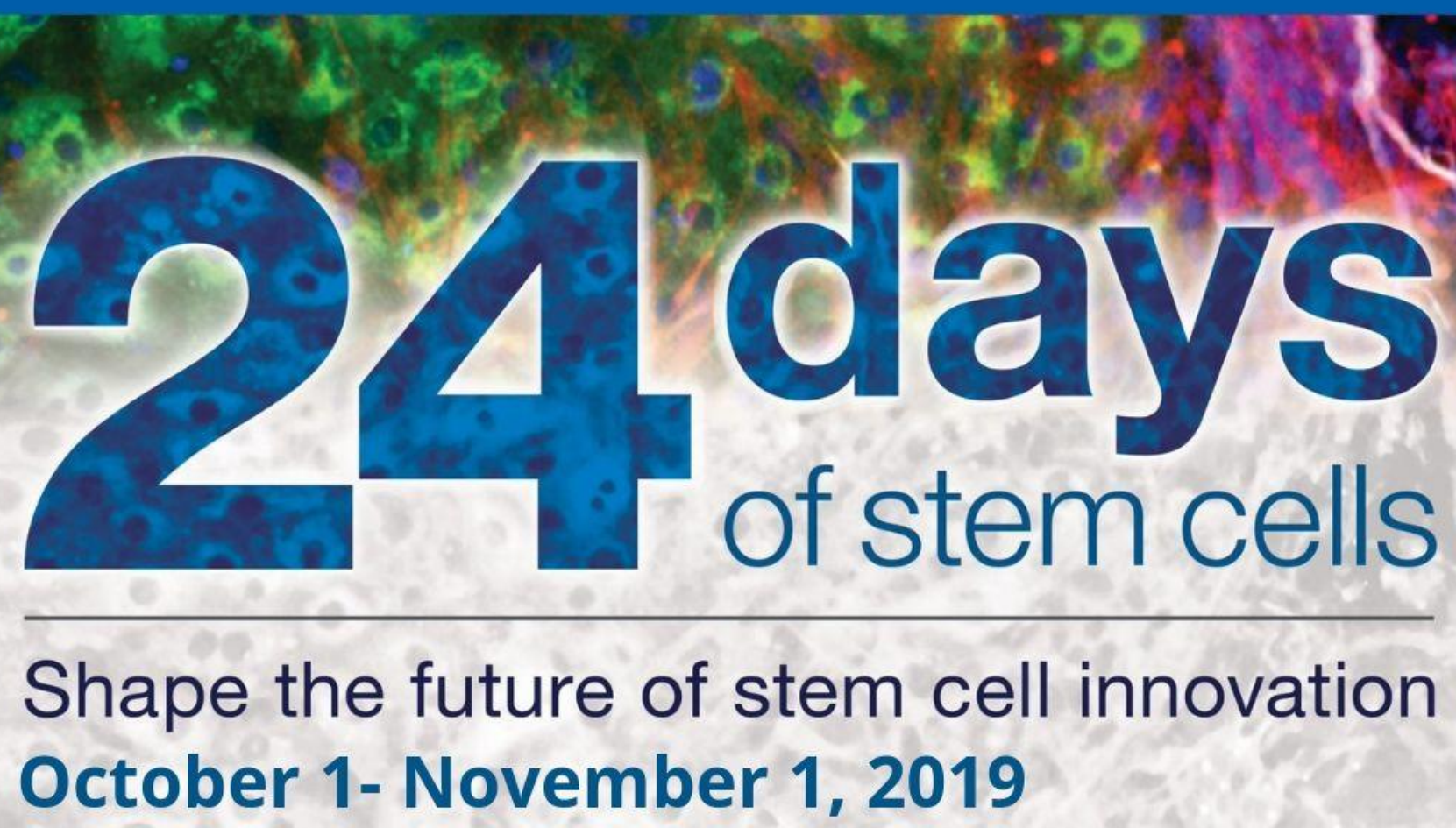

Join us for 24 Days of Stem Cells; a premiere virtual event featuring the latest advances in stem cell research.

This year's format will feature a new hour of cutting edge content every week day starting October 1st. Attend the sessions that are most relevant to your work - at your convenience and at your pace.

During the 24-day long event, you can:

- Access leading scientific presentations from thought leaders around the world

- Watch live training demonstrations from our stem cell experts

- Download key stem cell tools and resources

- Complete weekly challenges to earn points towards certification and prizes

Register today at

ThermoFisher www.24daysofstemcells.com S C I E N T I F I C 


\title{
Soluble adenylyl cyclase-mediated cAMP signaling and the putative role of PKA and EPAC in cerebral mitochondrial function
}

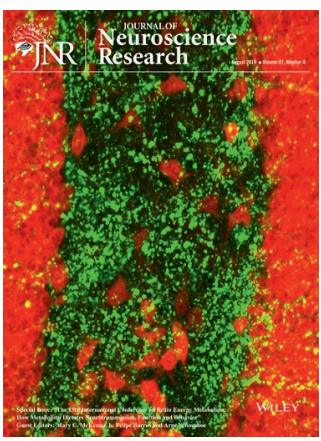

\author{
Emil Jakobsen (D) | Sofie C. Lange | Lasse K. Bak
}

Faculty of Health and Medical Sciences, Department of Drug Design and

Pharmacology, University of Copenhagen, Copenhagen, Denmark

\section{Correspondence}

Lasse K. Bak, Faculty of Health and Medical Sciences, Department of Drug Design and Pharmacology, University of Copenhagen, Universitetsparken 2, Copenhagen 2100, Denmark.

Email: Laba@sund.ku.dk

\section{Funding information}

Lundbeckfonden, Grant/Award Number: R249-2017-109; Hørslev-Fonden, Grant/ Award Number: N/A; Augustinus Fonden, Grant/Award Number: N/A

\begin{abstract}
Mitochondria produce the bulk of the ATP in most cells, including brain cells. Regulating this complex machinery to match the energetic needs of the cell is a complicated process that we have yet to understand in its entirety. In this context, $3^{\prime}, 5^{\prime}$ cyclic AMP (cAMP) has been suggested to play a seminal role in signaling-metabolism coupling and regulation of mitochondrial ATP production. In cells, cAMP signals may affect mitochondria from the cytosolic side but more recently, a cAMP signal produced within the matrix of mitochondria by soluble adenylyl cyclase (SAC) has been suggested to regulate respiration and thus ATP production. However, little is known about these processes in brain mitochondria, and the effectors of the CAMP signal generated within the matrix are not completely clear since both protein kinase $A$ (PKA) and exchange protein activated by CAMP 1 (EPAC1) have been suggested to be involved. Here, we review the current knowledge and relate it to brain mitochondria. Further, based on measurements of respiration, membrane potential, and ATP production in isolated mouse brain cortical mitochondria we show that inhibitors of SAC, PKA, or EPAC affect mitochondrial function in distinct ways. In conclusion, we suggest that brain mitochondria do regulate their function via SAC-mediated CAMP signals and that both PKA and EPAC could be involved downstream of SAC. Finally, due to the role of faulty mitochondrial function in a range of neurological diseases, we expect that the function of SAC-CAMP-PKA/EPAC signaling in brain mitochondria will likely attract further attention.
\end{abstract}

\section{KEYWORDS}

cAMP, EPAC, mitochondria, OXPHOS, PKA, RRID:SCR_002798, RRID:SCR_014526, soluble adenylyl cyclase

\section{1 | INTRODUCTION}

This paper is part of a Journal of Neuroscience Research special issue published following the International Conference on Brain
Energy Metabolism hosted by Felipe Barros in Valdivia, Chile, in March 2018. The aim of the paper is to draw attention to the controversy on how intramitochondrial 3',5'-cyclic adenosine monophosphate (cAMP) regulates oxidative phosphorylation (OXPHOS), with a 


\section{Significance}

Mitochondrial function is compromised in a range of neurological diseases. SAC-mediated CAMP signaling has been suggested to regulate OXPHOS and thus SAC signaling may be a putative target for novel pharmacotherapeutic approaches to restore mitochondrial function. However, sAC signaling is severely understudied in relation to brain mitochondria. With this paper, we encourage further studies into the functional role of $\mathrm{SAC}$ signaling and its downstream effectors, PKA and EPAC1, in brain mitochondria in health and disease.

specific focus on neurometabolism. To fulfill this aim, this paper is a combined review and original paper; hence, the Introduction reviews the literature on how intramitochondrial CAMP regulates OXPHOS, while the experimental work provides pharmacological results that together with the discussion aim to convince peers within the field that this controversy deserves to be investigated more thoroughly in brain mitochondria.

It is estimated that during rest the human brain consumes approximately $20 \%$ of total body energy consumption (Mink, Blumenschine, \& Adams, 1981). Furthermore, it has been estimated that $70 \%-80 \%$ of the brain's energy consumption is related to neuronal signaling (Harris, Jolivet, \& Attwell, 2012). This high energy demand in relation to neuronal signaling suggests that mitochondria in active neurons must be capable of upregulating OXPHOS rapidly during neuronal signaling (Howarth, Gleeson, \& Attwell, 2012). Furthermore, mitochondrial dysfunction has been associated with several neurodegenerative diseases (Correia et al., 2012). Hence, understanding the mechanisms of how mitochondria regulate OXPHOS is crucial to obtain an in-depth understanding of the complexity of neurometabolism. Over the last decade, it has become evident that the mitochondrial matrix contains a distinct cAMP microdomain where CAMP is produced by soluble adenylyl cyclase (sAC; Acin-Perez, Salazar, Kamenetsky, et al., 2009; Di Benedetto, Gerbino, \& Lefkimmiatis, 2018). Several groups have reported that intramitochondrial CAMP plays a role in regulating OXPHOS; however, there is a controversy in the literature on whether this effect is mediated via protein kinase $A$ (PKA) or exchange protein directly activated by cAMP (EPAC) (e.g., Acin-Perez, Gatti, Bai, \& Manfredi, 2011; Acin-Perez, Salazar, Kamenetsky, et al., 2009; Covian, French, Kusnetz, \& Balaban, 2014; Wang et al., 2016).

\section{1 | cAMP signaling and compartmentalization}

The ubiquitous second messenger, cAMP, produced by adenylyl cyclase $(A C)$, is known to be involved in regulating numerous cellular functions including, but not limited to, gene transcription, cell migration, cell death, and cell metabolism (Horvat \& Vardjan, 2018; Lefkimmiatis \& Zaccolo, 2014). The variety of cAMP responses led Buxon and Brunton to suggest that cAMP functions in independent microdomains, an idea that is now well established (Buxton \& Brunton, 1983; Cooper, 2005; Scott, Dessauer, \& Taskén, 2013).

The first step to understanding cAMP microdomains starts with the variety of ACs. There are nine transmembrane ACs (tmAC 1-9) which respond to internal stimuli such as $\mathrm{Ca}^{2+}$ signaling as well as receptor-mediated external stimuli (Cooper \& Tabbasum, 2014). In contrast to the membrane-bound $\mathrm{ACs}, \mathrm{SAC}$ is found throughout the cytosol, as well as associated with or inside several organelles including mitochondria and the nucleus (Steegborn, 2014; Wiggins, Steegborn, Levin, \& Buck, 2018). sAC activity is regulated by bicarbonate, $\mathrm{Ca}^{2+}$, and ATP allowing SAC to respond to several cellular events including fluctuations in ATP levels or energy metabolism (since $\mathrm{CO}_{2}$ produced from TCA cycle activity rapidly is converted to bicarbonate via carbonic anhydrase) as well as playing a role in $\mathrm{Ca}^{2+} /$ cAMP cross-signaling (Buck \& Levin, 2011; Chen et al., 2000; Litvin, Kamenetsky, Zarifyan, Buck, \& Levin, 2003; Steegborn, 2014; Steegborn, Litvin, Levin, Buck, \& Wu, 2005).

cAMP has four known classes of effectors, PKA, EPAC, cyclic nucleotide-gated channels, and the Popeye domain-containing protein, of which the first two are of interest in this paper. Both PKA and EPAC are found throughout the cell and in association with or within several organelles (Cheng, Ji, Tsalkova, \& Mei, 2008; Lefkimmiatis \& Zaccolo, 2014). PKA, believed to be the major effector of CAMP, is a serine/threonine kinase (Lefkimmiatis \& Zaccolo, 2014) and is composed of two catalytic subunits and two regulatory subunits, the latter binding CAMP which releases the constitutively active catalytic subunits (Cheng et al., 2008). PKA is divided into two classes, PKA(I) and PKA(II) with varieties in the regulatory subunits (Cheng et al., 2008). Since its discovery two decades ago, EPAC has been associated with an increasing number of cAMP-mediated signaling pathways (Cheng et al., 2008; Schmidt, Dekker, \& Maarsingh, 2013). When activated by cAMP, EPAC favors GDP/GTP exchange and thereby activates Rap1 and Rap2 that in the cytosol may lead to downstream $I_{3} /$ $\mathrm{Ca}^{2+}$ signaling (Schmidt et al., 2013). There are two known isoforms of EPAC (EPAC1 and EPAC2) and both are widely distributed across tissues and cell types (Schmidt et al., 2013). cAMP is degraded to AMP by phosphodiesterases (PDEs), a group of enzymes consisting of 11 families and almost 100 isoforms (Brescia \& Zaccolo, 2016; Kokkonen \& Kass, 2017). Thus, cAMP signaling is terminated by PDE activity, and the many different isoforms, many with distinct activators or inhibitors, allow for creating great complexity and specific CAMP signaling domains depending on the PDEs expressed and/or located to a specific cAMP microdomain. In order to form tightly regulated CAMP micodomains, the CAMP producing ACs, cAMP effectors, and CAMP degrading PDEs must be kept in close spatial connection. A-kinase anchoring proteins (AKAPs) have been found throughout the cell and work as scaffolding proteins capable of gathering some or all of the abovementioned proteins into cAMP microdomains or, more accurately, nanodomains (Dessauer, 2009; Dodge-Kafka et al., 2005; Kritzer, Li, Dodge-Kafka, \& Kapiloff, 2012; Theurkauf \& Vallee, 1982). Currently, more than 50 AKAPs are known although only a handful 
have been studied in detail (see Figure 1a for a cartoon overview of cAMP signaling; Dessauer, 2009; Kritzer et al., 2012).

\section{2 | Mitochondrial cAMP microdomain(s)}

In mammalian cells, the outer mitochondrial membrane (OMM) is cAMP permeable, while the inner mitochondrial membrane (IMM) is cAMP impermeable (except when mitochondrial permeability transition (MPT) is induced); as an interesting contrast to this, the IMM in Drosophila has repeatedly been shown to be cAMP permeable (AcinPerez, Salazar, Kamenetsky, et al., 2009; Di Benedetto et al., 2018; Di Benedetto, Scalzotto, Mongillo, \& Pozzan, 2013; Lefkimmiatis, Leronni, \& Hofer, 2013; Zhang et al., 2015). It has been suggested by Acin-Perez and colleagues, and later verified by several others, that the mitochondrial matrix contains $S A C$ allowing for a mitochondrial matrix CAMP signaling domain to exist independently of cytosolic cAMP signaling (Acin-Perez, Salazar, Kamenetsky, et al., 2009; Di Benedetto et al., 2013; Valsecchi, Konrad, \& Manfredi, 2014; Zippin et al., 2003). Thus, when considering mitochondrial cAMP signaling, it is important to distinguish between the three different CAMP domains, mitochondrial matrix, the intermembrane space (IMS), and the OMM (Di Benedetto et al., 2018; Monterisi \& Zaccolo, 2017).

In support of the existence of cAMP signaling domains in mitochondria, the presence of PKA and possible proteins targeted by PKA in the mitochondrial matrix, IMS, and OMM have been reported (reviewed in Di Benedetto et al., 2018; Monterisi \& Zaccolo, 2017). These findings are in line with several studies pointing to a large number of possible PKA phosphorylation targets in mitochondria including complex IV (Figure 1b; Grimsrud et al., 2012; Zhao et al., 2010). To regulate CAMP-PKA signaling in mitochondria, several AKAPs have been reported in mitochondria, most of them associated with the OMM and IMM; however, one specific AKAP, sphingosine kinase interacting protein (SKIP), was also found in the mitochondrial matrix (as reviewed in Monterisi \& Zaccolo, 2017; Valsecchi, RamosEspiritu, Buck, Levin, \& Manfredi, 2013)). More recently, EPAC1 has also been identified in the mitochondrial matrix, suggesting that matrix CAMP signaling is affecting several downstream pathways with one suggested target of EPAC1 being the mitochondrial calcium uniporter (MCU) (Figure 1b; Qiao, Mei, Popov, Vergara, \& Cheng, 2002; Wang et al., 2016; Wiggins et al., 2018). PDEA2 has been suggested as a main terminator of cAMP signals in the mitochondrial matrix; however, other PDEs are likely to play a role (Acin-Perez, Russwurm, et al., 2011).

In summary, it seems evident that mitochondria may contain several complete and highly regulated CAMP signaling pathways, including one localized entirely to the matrix (see Figure $1 \mathrm{~b}$ for cartoon overview of cAMP signaling in the mitochondrial matrix).

\section{3 | The role of mitochondrial matrix cAMP signaling in regulating OXPHOS}

While there is consensus that the CAMP pool in the mitochondrial matrix is produced by SAC and is separated from the CAMP pool in the cytosol, the downstream effects of this cAMP signaling is highly debated with several studies suggesting opposite effects (Acin-Perez, Salazar, Kamenetsky, et al., 2009; Covian et al., 2014; Wang et al., 2016). Part of this disagreement might be caused by a high variety in the experimental approaches employed. The effect of mitochondrial matrix cAMP has been studied in vitro on isolated proteins, in isolated mitochondria, and in intact cells, all from a variety of animal and tissue origins. In the following section, focus will be on suggested roles of mitochondrial matrix CAMP-mediated regulation of OXPHOS. (a)

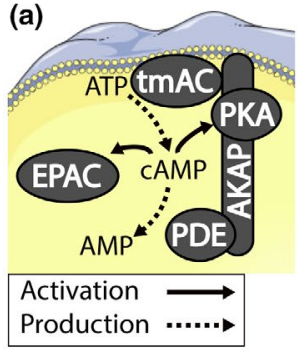

(b)

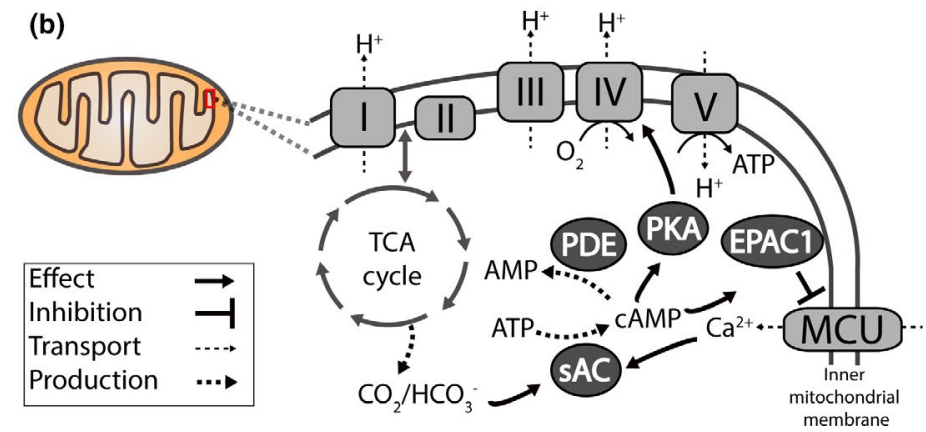

FIG URE 1 cAMP signaling and compartmentalization. (a) Cartoon of cAMP signaling depicting how cAMP is produced by AC (of which 10 isoforms exist, 9 of them are transmembrane bound; tmAC), degraded by PDE (of which almost 100 isoforms exist) as well as the two cAMP effectors PKA (which is divided into two classes) and EPAC (of which two isoforms exist). All of these can be kept in close spatial connection by the scaffolding protein AKAP of which more than 50 types are known. Some AKAPs are capable of composing highly complex compositions of ACs, effectors, and PDEs to create a specific cAMP microdomain. Cell template is used from http://smart.servier.com/. (b) Cartoon of a mitochondrial matrix cAMP domain. The inner mitochondrial membrane in impermeable to cAMP, thus intramitochondrial CAMP signals are produced by intramitochondrial SAC. SAC is activated by bicarbonate, which is in constant equilibrium with $\mathrm{CO}_{2}$ produced in the TCA cycle, and by $\mathrm{Ca}^{2+}$. PDEA2 has been identified in the mitochondrial matrix, but other unidentified PDEs are believed to exist in the matrix as well. The cAMP effector PKA has been suggested in the mitochondrial matrix, along with several possible PKA phosphotargets, one of these is Complex IV of the ETC. The CAMP effector EPAC has also been identified in the mitochondrial matrix. At this point $\mathrm{MCU}$ is the only suggested downstream target of EPAC in the matrix. Both cartoons are based on references found in the introduction 
In 2009, Acin-Perez and colleagues suggested a $\mathrm{CO}_{2}-\mathrm{HCO}_{3}{ }^{-}$-sACcAMP-PKA-complex IV signaling pathway within the mitochondrial matrix based on experiments in isolated mitochondria from mouse liver and HeLa cells (Acin-Perez, Salazar, Kamenetsky, et al., 2009). The pathway was suggested to be initiated by $\mathrm{CO}_{2}$ produced by the TCA cycle which leads to phosphorylation of a complex IV target increasing respiratory capacity, ATP production, and activity of the electron transport chain (ETC) complexes I, II, and IV (Acin-Perez, Salazar, Kamenetsky, et al., 2009). In a series of follow-up studies, the same group suggested that the pathway was active in isolated mitochondria from mouse brain (Acin-Perez, Gatti, et al., 2011; Acin-Perez, Russwurm, et al., 2011; Acin-Perez, Salazar, Brosel, et al., 2009). Furthermore, in mouse fibroblast cells and in mouse liver mitochondria they identified Ser58 of Complex IV-subunit 1 as the PKA target, a residue that is conserved among mammals; the phosphorylation prevents ATP inhibition of complex IV. Lastly, it was suggested that PDE2A is regulating CAMP levels in liver mitochondria, and to some extent in brain mitochondria (Acin-Perez, Russwurm, et al., 2011). Later, in 2013, Di Benedetto and colleagues (2013) confirmed that the mitochondrial matrix contained SAC using a variety of pharmacological and genetic tools (siRNA silencing and overexpression). The link between SAC activity and ATP production was also verified, and PKA was suggested as the CAMP effector in this pathway; however, contrary to previous reports, they could not increase ATP production with two different PKA agonists. Lastly, it was shown in primary cultured neonatal rat cardiomyocytes that $\mathrm{Ca}^{2+}$ signaling plays an important role in regulating mitochondrial matrix cAMP levels. In 2016, Ramos-Espiritu and colleagues showed that inhibition of SAC in mouse embryo fibroblasts led to a decrease in complex IV activity, a decrease that was not observed in SAC knockout (KO) cells (Ramos-Espiritu et al., 2016). One year later, Valsecchi and colleagues performed a very elegant study to distinguish the roles of cytosolic and mitochondrial SAC in the same model system. In SAC KO cells, complex I and complex IV activity as well as ATP production, coupled respiration, and membrane potential were hampered, and expression of some OXPHOS enzymes were increased. All of these factors were normalized when $\mathrm{SAC}$ was expressed in the mitochondria. Cytosolic SAC KO caused changes in $\mathrm{IP}_{3} \mathrm{R}$ signaling which led to changes in $\mathrm{ER} \mathrm{Ca}^{2+}$ release (Valsecchi et al., 2017). Recently, in a pharmacological characterization of the SAC inhibitors 2-OHE, KH7 and bithionol, we showed that inhibition of SAC in cerebral cortical-isolated mitochondria caused decreased ATP production, respiration and membrane potential thus confirming the presence of the SAC pathway in brain mitochondria (Jakobsen et al., 2018).

While the work listed above seem to have verified a clear model describing a link between mitochondrial matrix cAMP produced by SAC and OXHPOS, mediated via PKA, other recent studies seem to disagree with the proposed model. In 2013, Lefkimmiatis and colleagues verified in HeLA cells that CAMP does not permeate the $I M M$, and that bicarbonate increased CAMP levels in the matrix (Lefkimmiatis et al., 2013). However, no PKA activity was observed in HeLa or HEK cells, when treated with bicarbonate or a PKA agonist (Lefkimmiatis et al., 2013). Similar results were obtained by Burdyga and colleagues who observed no CAMP/PKA-mediated phosphorylation in the matrix or IMS but only at the OMM in neonatal rat ventricular myocytes (NRVM) and HeLa cells (Burdyga et al., 2018). Furthermore, Monterisi and colleagues found no presence of PDE2A in the mitochondrial matrix, but only at the IMM and OMM in NRVM and HeLa cells (Monterisi et al., 2017). In 2016, Wang and colleagues verified in isolated rat heart mitochondria that SAC produces CAMP in the matrix. The cAMP signal induced increased membrane potential, respiration and ATP production; the latter was also induced by $\mathrm{Ca}^{2+}$ (Wang et al., 2016). When MPT was induced with $10 \mu \mathrm{M} \mathrm{Ca}^{2+}$, SAC activation by bicarbonate slowed the rate of loss in membrane potential, while inhibition of SAC increased the rate (Wang et al., 2016). This observation led the authors to suggest that SAC-produced CAMP regulates the $\mathrm{MCU}$, which was found to be mediated by the CAMP effector EPAC1, and importantly was independent of PKA activity (Wang et al., 2016). Furthermore, it was observed that the EPAC1 inhibitor CE3F4 not only decreased basal respiration but also eliminated the $\mathrm{HCO}_{3}{ }^{-}$-induced increase in respiration (Wang et al., 2016). These findings led the authors to suggest that EPAC1 is also involved in regulating OXPHOS in mitochondria (Wang et al., 2016). Further, in 2014, Covian and colleagues (2014) saw no effect on respiration in pig heart isolated mitochondria when stimulating or inhibiting PKA; however, $\mathrm{Ca}^{2+}$ increased the respiration twofold, even in the presence of a PKA inhibitor. In contrast to other studies, they found that the CAMP analog 8-CPT-6-PhecAMP inhibited mitochondrial respiration by decreasing the flow of electrons entering ETC, which they argued was due to the lipophilic characteristics of the molecule. Furthermore, the study revealed no PKA activity in solubilized mitochondria, even in the presence of PKA agonists, nor could any PKA subunits be identified in the mitochondria using mass spectrometry. In contrast to this observation, Agnes and colleagues showed in isolated mitochondria from bovine heart that $85 \%$ of mitochondrial PKA was found in the mitochondrial matrix (Agnes, Jernigan, Shell, Sharma, \& Lawrence, 2010).

As is evident from the above, there is controversy in relation to how CAMP produced in the mitochondrial matrix might affect OXPHOS. Some of these differences could be related to the variance in origin of the cells and mitochondria in which this hypothesis has been tested. Both Covian et al. (2014) and Wang et al. (2016) found no evidence of PKA activity in heart mitochondria from rat and pig, respectively, while Acin-Perez found that OXPHOS in isolated mitochondria from rat and mouse liver and brain were regulated by PKA (Acin-Perez, Russwurm, et al., 2011; Acin-Perez, Salazar, Brosel, et al., 2009; Acin-Perez, Salazar, Kamenetsky, et al., 2009). Covian et al. (2014) argues that the "heart has a significant larger range of ATP turnover than the liver or cultured cells," and that this might explain the observed differences in regulation of OXPHOS. In line with this argument, mitochondrial heterogeneity in terms of protein expression between different organs has previously been reported, and could possibly account for some of the observed differences (Johnson, Harris, Blair, \& Balaban, 2007; Johnson, Harris, French, et al., 2007). However, opposite results, for example, observed in HeLa and HEK cells by Acin-Perez, Salazar, Kamenetsky, et al. (2009) and Lefkimmiatis et al. (2013) might be induced by differences in the 
experimental design as suggested in the review by Di Benedetto et al. (2018).

In summary, the mitochondrial matrix contains a distinct cAMP domain, which functions independently of cytosolic cAMP. Intramitochondrially produced CAMP likely plays a role in regulating OXPHOS. However, the downstream target of CAMP seems to be variable in mitochondria originating from different tissues. The brain is a highly energy-demanding organ, and the function and regulation of cerebral mitochondria may differ from mitochondria in other tissues. To further elucidate and consolidate the role of SAC, PKA, and EPAC1 in brain energy metabolism, we tested the effects of a variety of SAC, PKA and EPAC1 inhibitors on respiration, ATP production and membrane potential in isolated mouse cortical mitochondria.

\section{2 | METHODS}

\section{1 | Materials}

Adenosine-5'-diphosphate (ADP, A5285), adenosine-5'-triphosphate (ATP, A8937), antimycin A (A8674), carbonyl cyanide 4-(trifluoromethoxy)phenylhydrazone (FCCP, C2920), H89 (B1427), LRE-1 (SML1857), malic acid (M1000), oligomycin A (75351), P1,P5-Di(adenosine-5') pentaphosphate pentasodium (D4022), pyruvic acid (107360), and tetramethylrhodamine methyl ester (TMRM, T5428) were purchased from Sigma-Aldrich (St. Louis, MO, USA). CE3F4 (4793) and PKI 14-22 amide, myristoylated (2546) were purchased from Tocris (Lille, France). EPAC 5376753 (10-1564) was from Focus Biomolecules (Plymouth Meeting, PA, USA). PercolI ${ }^{\mathrm{TM}}$ (17-0891-01) was from GE Healthcare (Uppsala, Sweden. Luciferin-luciferase (11-501) was from BioThema $A B$ (Handen, Sweden) and used according to the manufacturer's instructions. All other chemicals were of appropriate purity and obtained from commercial sources.

\section{2 | Animals}

Male NMRI mice (Envigo, Cambridgeshire, United Kingdom) aged 8-9 weeks were housed in a pathogen-free, temperature and humidity-controlled environment at the Department of Drug Design and Pharmacology, University of Copenhagen. Mice were handled according to standards given by the Danish Animal Experiments Inspectorate. The mice were housed in groups of four in individually ventilated cages with free access to chow and water. Mitochondrial function is affected by sex steroids and the SAC inhibitor 2-hydroxy estradiol is an estrogen derivative (Gaignard et al., 2017; Steegborn, Litvin, Hess, et al., 2005). Thus, in order to avoid any potential effects of hormones from female mice being in different stages of the estrous cycle, all experiments were performed in male mice, and hence sex-specific aspects of the work could not be evaluated.

\section{3 | Isolation of brain cortex mitochondria}

The isolation procedure was performed on ice or at $4^{\circ} \mathrm{C}$ as previously described (Andersen, Christensen, Nissen, \& Waagepetersen,
2017). Mice were euthanized by cervical dislocation, decapitated and the brain quickly excised from the cranial vault. Brain cortex was excised, transferred to isolation buffer (MSHE; in mM: mannitol 210, sucrose 70, HEPES 5, EGTA 1 , and 0.5\% BSA; $\mathrm{pH}=7.2$ ) and gently homogenized using a teflon-on-glass douncer at 500 revolutions/min for 7-8 strokes. The homogenate was centrifuged $(500 \mathrm{~g} \times 5 \mathrm{~min})$ to pellet cell remnants. The supernatant was centrifuged $(14,000 \mathrm{~g} \times 10 \mathrm{~min})$ and the pellet resuspended in $12 \%$ Percoll MSHE solution. This suspension was gently layered on a $21 \%$ Percoll MSHE solution and centrifuged $(18,000 \mathrm{~g} \times 15 \mathrm{~min})$. The supernatant was discarded and the pellet was washed with two subsequent centrifugations $(18,000 \mathrm{~g} \times 5 \mathrm{~min}$ and $14,000 \mathrm{~g} \times 5 \mathrm{~min}$ ) by suspending the pellet in MSHE buffer and discarding the supernatant after each centrifugation. The final pellet, containing the isolated mitochondria, was resuspended and diluted in MSHE and protein amounts were determined using the Bradford protein assay with BSA as the reference protein.

\section{4 | Mitochondrial respiration-Seahorse XFe96 assay}

Respiration of isolated brain cortex mitochondria was monitored using a Seahorse XFe96 instrument (Agilent, Santa Clara, USA) (Andersen et al., 2017). Mitochondria were isolated as described above, diluted to $80 \mu \mathrm{g}$ protein $/ \mathrm{ml}$ in respiration buffer (in $\mathrm{mM}$ : sucrose 250; $\mathrm{K}_{2} \mathrm{HPO}_{4} 15 ; \mathrm{MgSO}_{4} 2$; EDTA (free acid) 0.5 and BSA $0.5 \mathrm{~g} / \mathrm{L} ; \mathrm{pH} 7.2$ ) and $25 \mu \mathrm{l}$ was added to each well ( $2 \mu \mathrm{g}$ protein/ well) in a 96-well Seahorse plate. The plate was centrifuged $(1,500 \mathrm{~g}$ $\times 20 \mathrm{~min} \times 4^{\circ} \mathrm{C}$ ) and $155 \mu \mathrm{l}$ of respiration buffer containing respiratory substrates (final concentrations (f.c.) $10 \mathrm{mM}$ pyruvate and $2 \mathrm{mM}$ malate) and pharmacological compounds, as described, were added to each well. The plate was transferred to the XFe96 instrument and the experiment was conducted at $37^{\circ} \mathrm{C}$. Mitochondrial respiration was monitored as $\mathrm{pmol}_{2} / \mathrm{min}$. Each measurement cycle lasted $3 \mathrm{~min}$ and was followed by $2 \mathrm{~min}$ of mixing plus $1 \mathrm{~min}$ of rest. Each injection was followed by 1 min of mixing before the 3 min measurement cycle. After three baseline measurement cycles, ADP (potassium salt, f.c. 4 mM), oligomycin A (f.c. $2.5 \mu \mathrm{g}$ / $\mathrm{ml}$; blocking mitochondrial complex $\mathrm{V}$ and thus ATP production leaving only respiration not driven by ATP synthesis), FCCP (f.c. $4 \mu \mathrm{M}$; uncouples respiration from ATP synthesis and causes nearmaximal respiration), and antimycin A (f.c. $4 \mu \mathrm{M}$; blocks transfer of electrons from mitochondrial complex III to IV and thus leaves only respiration not caused by mitochondria) were injected with a measurement cycle following each injection. Two additional measure cycles were performed after the addition of antimycin A to determine the non-mitochondrial respiration. Data were collected and extracted using the Wave 2.6.0 software (Agilent, Santa Clara, CA, USA; RRID:SCR_014526). All conditions were performed with four technical replicates. Basal respiration, state 3 respiration and state 3 uncoupled respiration ( $\mathrm{pmol} \mathrm{O}_{2}$ consumed/min) for each pharmacological condition were normalized to control and presented as \% of control. Proton leak was calculated as the 
difference between respiration after injection of oligomycin $A$ and respiration after injection of antimycin $A$, and presented as \% of control. Respiration control ratio (RCR) was calculated as: (state 3 respiration-respiration after antimycin A)/proton leak.

\section{5 | Mitochondrial ATP synthesis-Luciferin- luciferase assay}

The procedure is modified from Lanza and Nair (2009) and Manfredi, Yang, Gajewski, and Mattiazzi (2002). Mitochondria were isolated as described above and diluted to $10 \mu \mathrm{g}$ protein/ $\mathrm{ml}$ in respiration buffer containing respiratory substrates $(10 \mathrm{mM}$ pyruvate, $2 \mathrm{mM}$ malate and $0.2 \mu \mathrm{M}$ P1,P5-Di(adenosine-5') pentaphosphate pentasodium; the latter was added to inhibit adenylate kinase activity) and pharmacological compounds where indicated. ATP production was monitored in a clear bottom 96well plate at $28^{\circ} \mathrm{C}$ (for optimal luciferin-luciferase response) in a NOVOstar microplate reader (BMG Labtech $\mathrm{GmbH}$, Ortenberg, Germany) from $2.5 \mu \mathrm{g}$ mitochondria by injection of $150 \mu \mathrm{l}$ luciferin-luciferase and $15 \mu \mathrm{l}$ hexokinase-purified ADP (potassium salt; to a final concentration of $0.6 \mathrm{mM}$ ) to each well and subsequent luminescence readings for $38 \mathrm{~s}$ followed by injection of $9.62 \mu \mathrm{M}$ ATP (f.c.; potassium salt) as internal standard. As a negative control, some wells were added $2.5 \mu \mathrm{g} / \mathrm{ml}$ oligomycin A, to inhibit ATP synthesis (data not shown). ATP production/(min $\times$ mg protein) in the presence of the compounds was normalized to the control and presented as \% of control. All conditions were performed with six technical replicates.

\section{6 | Mitochondrial membrane potential- TMRM assay}

Mitochondria were isolated as described above. Mitochondrial membrane potential $(\Delta \psi \mathrm{m})$ was monitored by TMRM $(1 \mu \mathrm{M})$ fluorescence in quench mode (i.e., the quenching, or decrease, in TMRM fluorescence upon uptake into the mitochondria is employed to approximate changes in the membrane potential) (Nicholls \& Ward, 2000) at $530 \mathrm{~nm}$ excitation and $590 \mathrm{~nm}$ emission after equilibration to a stable baseline at $28^{\circ} \mathrm{C}$ in a NOVOstar microplate reader (BMG Labtech $\mathrm{GmbH}$ ). Measurements were performed in a clear bottom 96-well plate in respiration buffer in the presence of respiratory substrates (10 $\mathrm{mM}$ pyruvate, $2 \mathrm{mM}$ malate, and $0.2 \mu \mathrm{M}$ P1,P5-Di(adenosine- $5^{\prime}$ ) pentaphosphate pentasodium plus $0.6 \mathrm{mM}$ ADP) with isolated mitochondria $(1 \mu \mathrm{g} /$ well) and addition of $10 \mu \mathrm{l}$ compound solutions to reach the final concentrations as indicated. After 31 min and 36 min oligomycin A $(5 \mu \mathrm{M})$ and FCCP $(4 \mu \mathrm{M})$ were added, respectively. Measurements of mitochondrial membrane potential are presented as one representative experiment for selected conditions. Area under the curve (AUC) was calculated for the interval 10-31 min using GraphPad Prism 7 (RRID:SCR_002798), and the conditions with pharmacological treatment were normalized to control and presented as \% of control. All conditions were performed with five technical replicates.

\subsection{Analysis and presentation of data}

Data shown represent means $\pm S D$ (unless otherwise noted), with individual data points plotted. Sample sizes were estimated based on previous experiments (Andersen, Jakobsen, Waagepetersen, \& Aldana, 2019; Jakobsen et al., 2018). For each assay, $\mathrm{n}$ equals the number of experiments where each individual experiment is equivalent to a single animal. Seahorse assays were performed as six individual experiments. For the ATP and membrane potential measurements, all conditions could not be carried out in a single experiment; thus, for each pharmacological condition five experiments were conducted (except for EPAC 53767535 MM ATP measurement, where only four experiments were conducted). Appropriate DMSO (solvent of inhibitors) controls were included and are presented as an individual data set. Since the DMSO controls were included in all experiments and are controls for all the analyzed compounds, the DMSO conditions were tested with more experiments than the pharmacological compounds. All data were analyzed employing GraphPad Prism 8.

All data points (except RCR values) were normalized to the respective controls and presented as \% of control. To evaluate the effect of DMSO, the DMSO data were compared to $100 \%$ (representing the normalized, blank samples) employing a one-sample $t$ test. This test can be used since the test variables are continuous and independent, are assumed to be normal distributed and since no outliers were found. The RCR values were not normalized to control since the absolute value of the RCR is of interest. These were compared to the raw RCR values of the control employing a oneway ANOVA with Dunnett's multiple comparisons posttest as were samples treated with pharmacological inhibitors (except $2 \mu \mathrm{M} \mathrm{PKI}$ which was the only compound containing $0.2 \%$ DMSO; data points from this condition were compared employing an unpaired $t$ test). Data from the pharmacological treatments are assumed to follow a normal distribution.

$p$ values $<0.05$ are considered significant and are indicated as follows: ${ }^{*}=p<0.05 ;{ }^{* *}=p<0.01 ;{ }^{* * *}=p<0.001 ;{ }^{* * * *}=p<0.0001$, $p$ values $<0.075$ are considered as trends. Means, standard deviations, number of biological replicates, and all statistical analyses are reported in the data tables (Tables 1-4).

\section{3 | RESULTS}

\subsection{Assessment of solvent effects}

Dimethyl sulfoxide (DMSO) was utilized to solubilize the pharmacological compounds employed in this study, causing final concentrations of DMSO to vary from $0.005 \%$ to $0.2 \%$. DMSO has been shown to affect metabolism in acute brain slices in concentrations as low as 0.000025\% (Nasrallah, Garner, Ball, \& Rae, 2008), and been suggested to modify OXPHOS, possibly by changing the kinetics of the ATP synthase (Conover, 1969; de Meis \& Suzano, 1988). Furthermore, DMSO has been shown to affect membrane permeability enhancement (Notman, Noro, O'Malley, \& Anwar, 2006). Thus, to assess the effect 


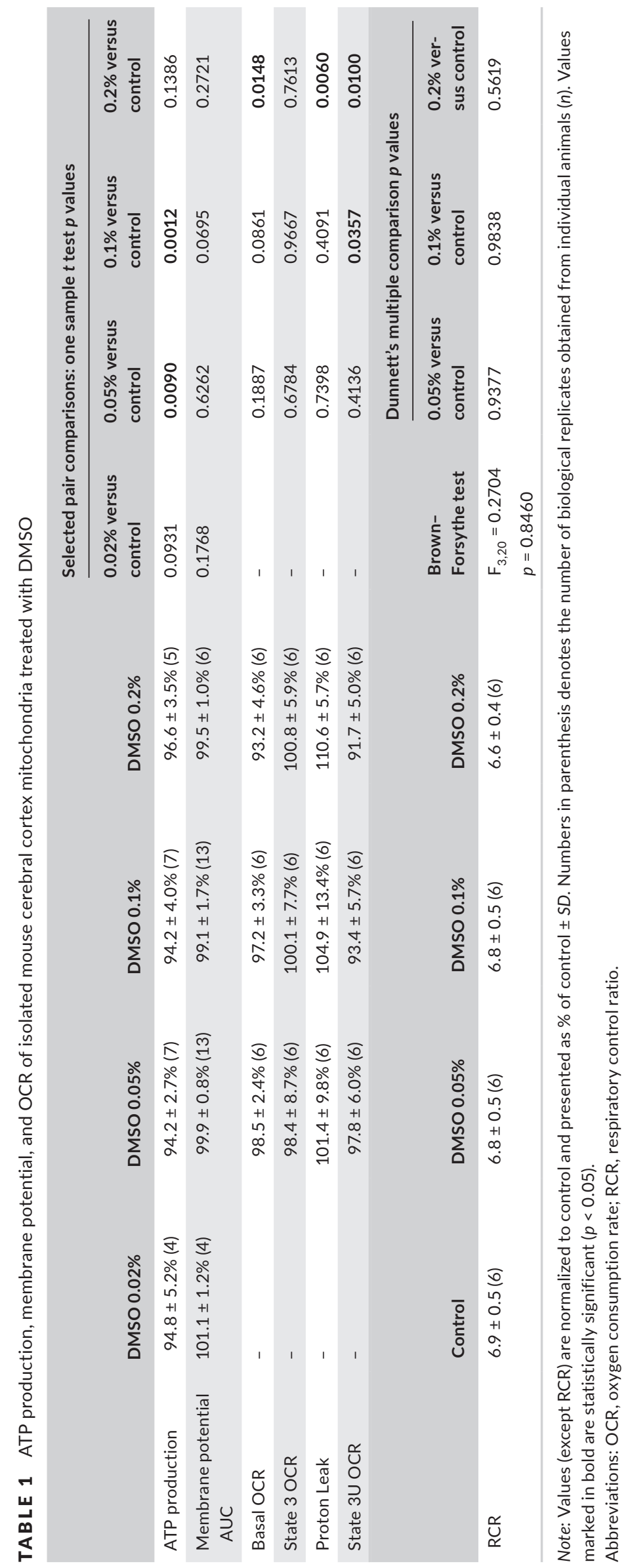




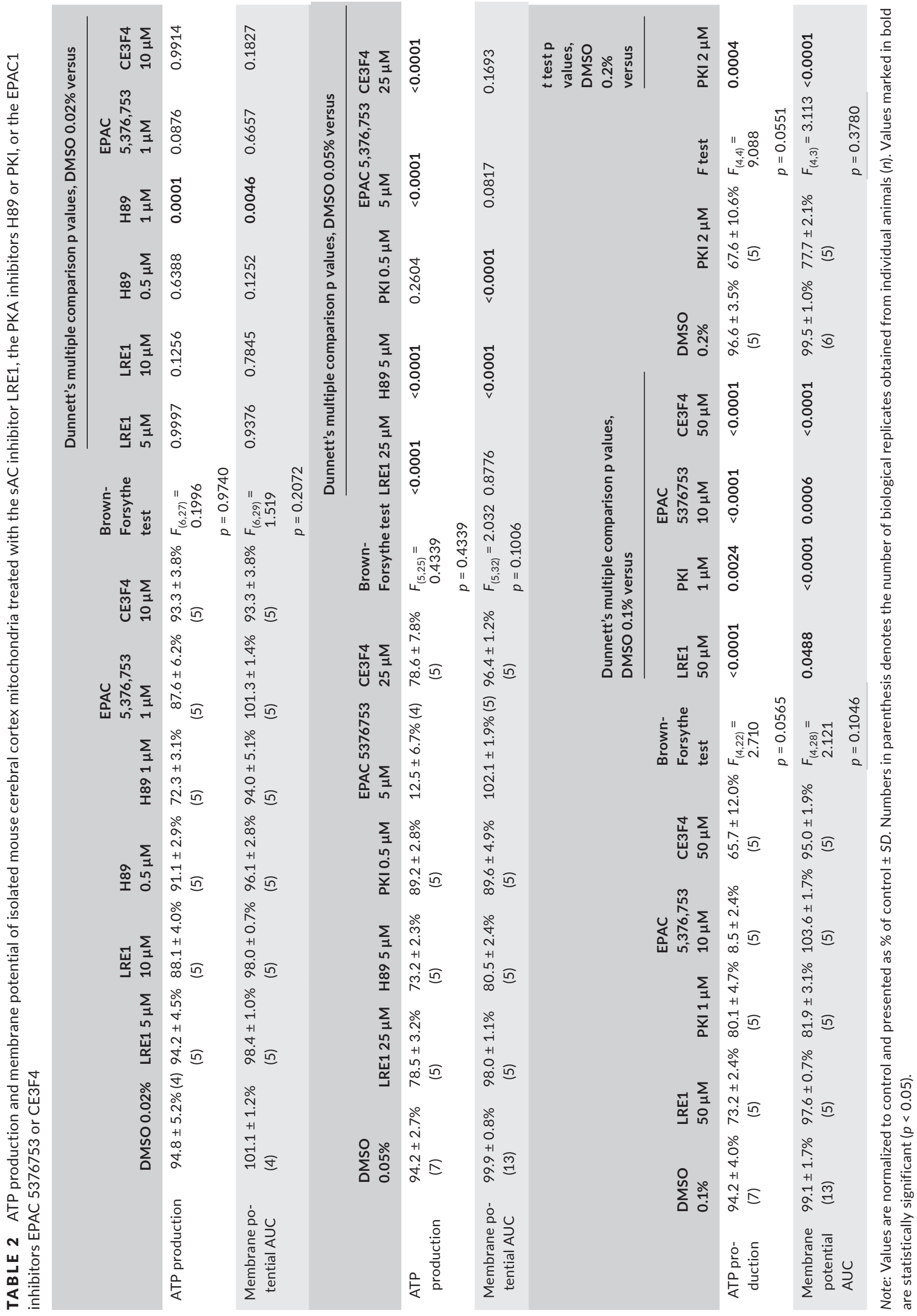




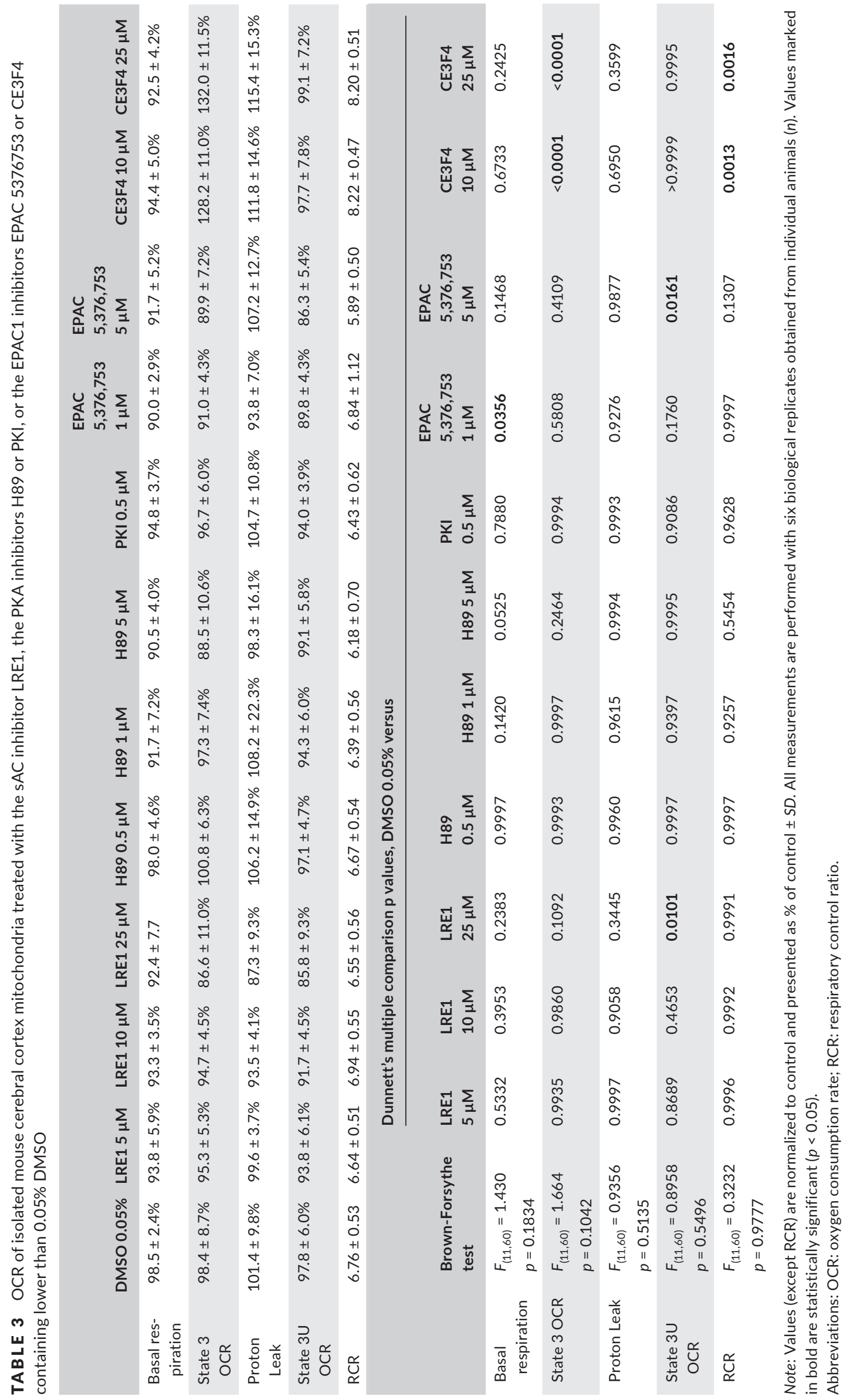




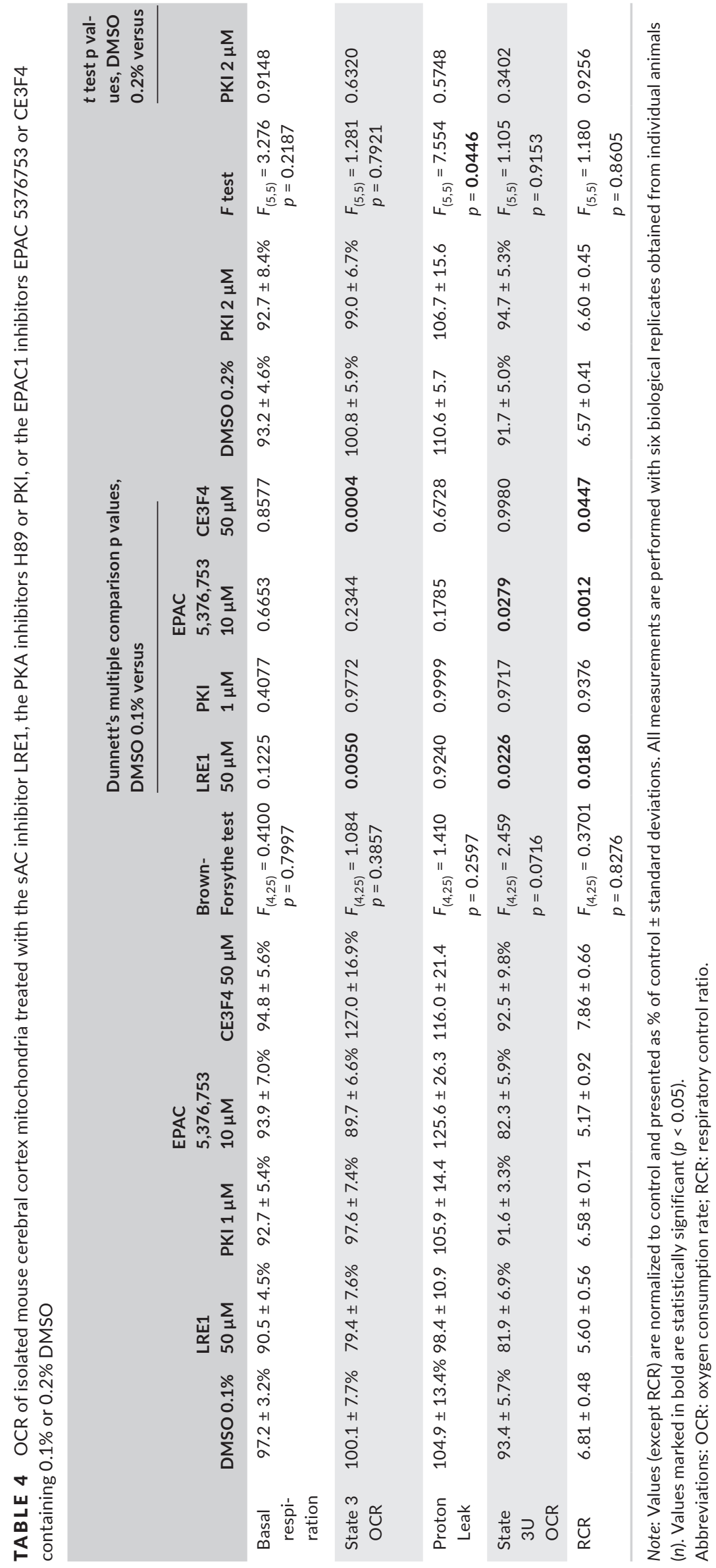


of DMSO on this preparation of cortical cerebral mitochondria, ATP production, oxygen consumption rate (OCR), and membrane potential were measured. DMSO caused a small decrease in ATP production to around $95 \%$ of control; however, only 0.05 and $0.1 \%$ DMSO were significantly lower than the control (Figure 2a; Table 1). Mitochondrial membrane potential was investigated with the dye TMRM in quench mode (see the Methods section), and DMSO (0.02\%, 0.05\%, $0.1 \%$ or $0.2 \%)$ had no effect on the membrane potential calculated as AUC $\left(\Delta \Psi_{\mathrm{m}}(\mathrm{FO} / \mathrm{F})\right)$ when compared to the control (Figure $2 \mathrm{~b},+\mathrm{C}$; Table 1$)$.

Finally, the OCR was investigated employing a Seahorse Extracellular Flux analyzer (see the Methods section). DMSO (0.2\%) significantly decreased baseline respiration to $93.2 \%$ of control (Figure 2d; Table 1). DMSO had no effect on state 3 respiration, but $0.2 \%$ caused a significant increase in proton leak to $110.6 \%$ of control (Figure 2e,+F; Table 1). DMSO (0.1 and 0.2\%) also induced a small decrease in state 3 uncoupled respiration (Figure $2 \mathrm{~g}$; Table 1). The respiratory control ratio was calculated to $6.89 \pm 0.5$ for the control and DMSO induced no change due to the concentrations tested (Figure $2 \mathrm{~h}$; Table 1 ). The observed effects of DMSO showed that it was necessary to take these into account when interpreting the effect of the pharmacological compounds employed; thus, all conditions tested where the drugs are dissolved in DMSO are compared to appropriate DMSO control. An overview of the DMSO concentration used in the different conditions can be found in Tables 2-4.

\section{2 | Inhibition of sAC decreases mitochondrial respiration and ATP production but have little effect on membrane potential}

Inhibition of mitochondrial SAC with a variety of different inhibi tors has been employed widely in the literature (e.g., Acin-Perez, Salazar, Kamenetsky, et al., 2009; Di Benedetto et al., 2013). We recently published a characterization of some of these inhibitors and based on this characterization we decided to employ the newly discovered inhibitor LRE-1 (Jakobsen et al., 2018; Ramos-Espiritu et al., 2016).

Inhibition of SAC with 5 or $10 \mu \mathrm{M}$ LRE1 had no effect on ATP production, while 25 and $50 \mu \mathrm{M}$ significantly decreased ATP production to $78.5 \%$ and $73.2 \%$ of control, respectively (Figure 3a; Table 2). LRE1 had a small effect on the membrane potential lowering it to around $98 \%$ of control. Despite the small numerical change, the decrease induced by $50 \mu \mathrm{M}$ was significantly lower than the respective DMSO control (Figure 3b,+C; Table 2). LRE1 had no significant effect on baseline OCR (Figure 3d; Tables 3,4). LRE1 (50 $\mu \mathrm{M})$ decreased state 3 OCR to $79.4 \%$ of control being significantly lower than the respective DMSO control (Figure 3e; Table 4), while having no significant effect on the proton leak (Figure 3f; Table 3,4). Furthermore, 25 and $50 \mu \mathrm{M}$ LRE1 significantly decreased state 3 uncoupled OCR to $85.8 \%$ and $81.9 \%$ of control, respectively (Figure $3 g$; Table 3,4). While LRE1 in concentrations of 5,10 , and $25 \mu \mathrm{M}$ had no effect on RCR, LRE1 $50 \mu \mathrm{M}$ significantly reduced RCR (Figure 3h; Tables 3,4).

\section{3 | Inhibition of PKA decreases ATP production and membrane potential but have no effect on respiration}

Several studies have suggested matrix PKA to play a downstream role in how SAC regulates OXPHOS (e.g., Acin-Perez, Salazar, Kamenetsky, et al., 2009; Di Benedetto et al., 2013). To investigate the role of PKA in regulating cortical cerebral mitochondrial function, the commonly used inhibitors of PKA, H89 (0.5, 1 or $5 \mu \mathrm{M})$, and $\mathrm{PKI}(0.5,1$ or $2 \mu \mathrm{M})$ were employed (Dalton \& Dewey, 2006; Lochner \& Moolman, 2006). H89 (0.5, 1 or $5 \mu$ M) decreased ATP production to $91.1 \%, 72.3 \%$, and $73.2 \%$ of control, respectively, the latter two being significantly lower than the respective DMSO controls (Figure 4a; Table 2). 1 and $5 \mu \mathrm{M}$ of H89 significantly decreased the membrane potential to 94.0 and $80.5 \%$ of control, respectively (Figure $4 b,+C$; Table 2 ).

H89 had no significant effect on basal OCR, however, $5 \mu \mathrm{M}$ showed a tendency toward decreased basal OCR (Figure 4d; Table 3). H89 showed no effect on state 3 OCR, proton leak, state 3 uncoupled OCR or RCR values (Figure $4 \mathrm{e}-\mathrm{h}$; Table 3 ).

$\operatorname{PKI}(0.5,1$, or $2 \mu \mathrm{M})$ decreased ATP production to $89.2 \%, 80.1 \%$, and $67.6 \%$ of control, respectively, the latter two being significantly lower than the respective DMSO controls (Figure 4a; Table 2). In terms of membrane potential, $\mathrm{PKI}(0.5,1$, and $2 \mu \mathrm{M})$ significantly decreased this to $89.6 \%, 81.9 \%$, and $77.7 \%$ of control, respectively (Figure $4 b,+C$; Table 2). PKI had no effect on basal OCR, state 3 OCR, proton leak, state 3 uncoupled OCR or RCR values (Figure 4d-h; Tables 3 and 4).

\subsection{Inhibition of EPAC1 causes dramatic decreases in ATP production with relatively smaller effects on respiration and membrane potential}

The cAMP effector, EPAC, has gained increased attention over the last two decades, and EPAC1 has been found inside mitochondria where it has been suggested to play a downstream role in how $\mathrm{SAC}$ regulates OXPHOS (Wang et al., 2016). To investigate the role of EPAC1 in cortical cerebral mitochondrial function, the inhibitors of EPAC1, EPAC 5376753, and CE3F4 were employed (Brown, Rogers, Aroonsakool, McCammon, \& Insel, 2014; Courilleau et al., 2012). EPAC 5376753 (

5 or $10 \mu \mathrm{M}$ ) significantly decreased ATP production to $12.5 \%$ and 8.5\% of control, respectively (Figure 5a; Table 2). EPAC 5376753 $(10 \mu \mathrm{M})$ slightly but significantly increased the membrane potential (Figure 5b,+C; Table 2). EPAC 5376753 slightly decreased basal OCR to $94 \%-90 \%$ of control but only the change induced by $1 \mu \mathrm{M}$ was significantly lower than the respective DMSO control (Figure $5 \mathrm{~d}$; Tables 3 and 4). Further, EPAC 5,376,753 had no effect on state 3 OCR or proton leak (Figure $5 \mathrm{e}-\mathrm{f}$; Tables 3 and 4 ). In terms of state 3 uncoupled OCR, EPAC $5,376,753$ (5 or $10 \mu \mathrm{M}$ ) significantly decreased the OCR to $86.3 \%$ and $82.3 \%$ of control (Figure $5 \mathrm{~g}$; Tables 3 and 4). 1 and $5 \mu \mathrm{M}$ EPAC 5,376,753 had no effect on RCR while $10 \mu \mathrm{M}$ significantly decreased RCR to 5.2 (Figure 5h; Tables 3 and 4).

CE3F4 $(10,25$, or $50 \mu \mathrm{M})$ decreased ATP production to $93.3 \%$, $78.6 \%$, and $65.6 \%$ of control, respectively, with the latter two being 
(a)

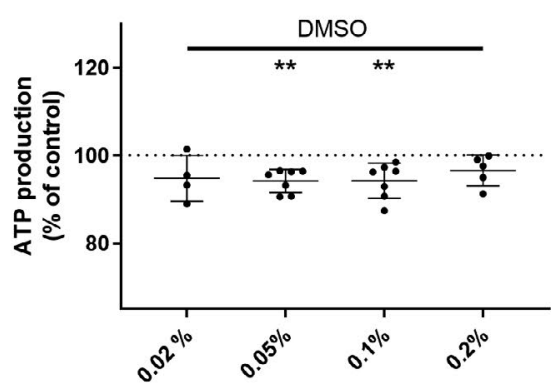

(c)

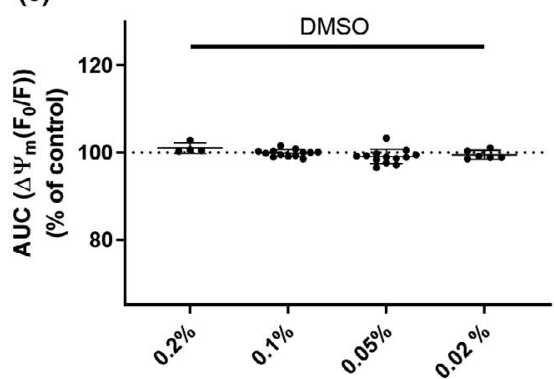

(e)

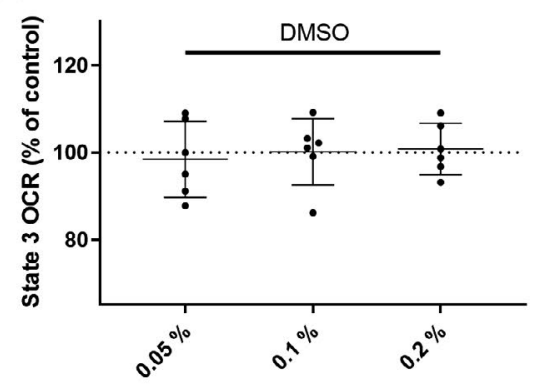

(g)

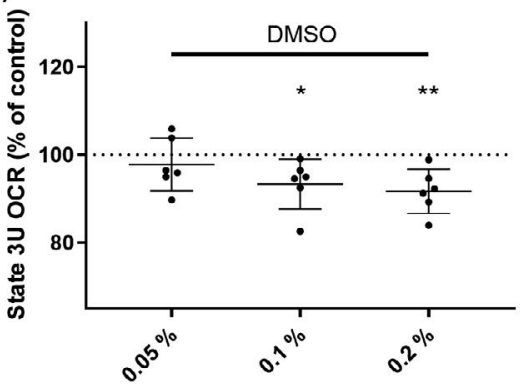

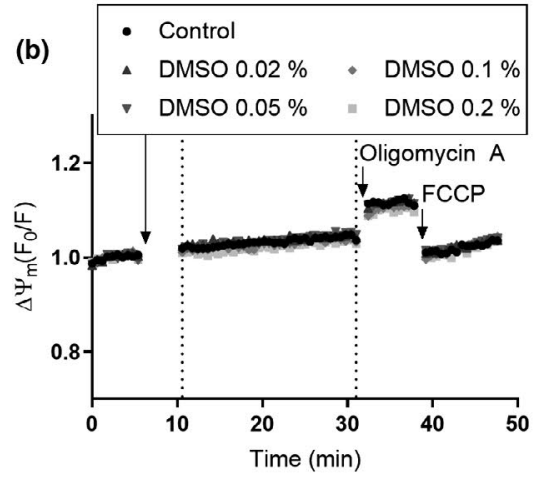

(d)
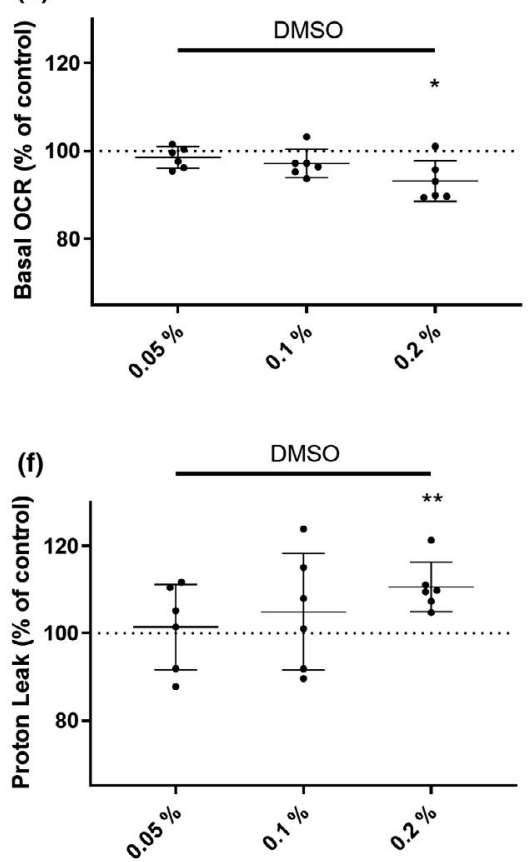

(h)

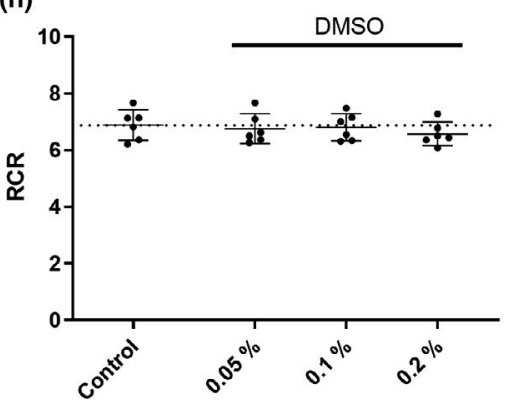

FIGURE 2 Assessment of solvent effects. Oxygen consumption, ATP production, and membrane potential were measured in isolated mitochondria of mouse cerebral cortex and challenged with the solvent DMSO $(0.02 \%, 0.05 \%, 0.1 \%$, or $0.2 \%)$ for ATP and membrane potential, and DMSO $(0.05 \%, 0.1 \%$, or $0.2 \%)$ for OCR measurements. Pyruvate $(10 \mathrm{mM})$ and malate $(2 \mathrm{mM})$ were utilized as substrates and all experiments were performed as described in the Methods section. (a) ATP production normalized to control; DMSO $0.02 \%, n=4 ; \mathrm{DMSO}$ $0.05 \%, n=7$; DMSO 0.1\%, $n=7$; DMSO $0.2 \%, n=5$. (b) Representative trace of membrane potential normalized to baseline measurements (0-5.4 min). Vehicle was added after $5.4 \mathrm{~min}$, oligomycin A were added after $31 \mathrm{~min}$, and FCCP were added after $36 \mathrm{~min}$. (c) AUCs of each trace in panel B were calculated in the interval from 10-31 min (indicated with dotted lines in panel B) normalized to control; DMSO 0.02\%, $n=4$; DMSO 0.05\%, $n=13$; DMSO 0.1\%, $n=13$; DMSO 0.2\%, $n=6$. (d) Basal respiration normalized to control; $n=6$ for all conditions. (e) State 3 respiration normalized to control; $n=6$ for all conditions. (f) Proton leak normalized to control; $n=6$ for all conditions. (g) State 3 uncoupled respiration normalized to control; $n=6$ for all conditions. (h) Respiratory control ratio (RCR); $n=6$ for all conditions. Statistics: panel a and c-g: one sample $t$ test Panel H: One-way ANOVA with Dunnett's multiple comparisons test; ${ }^{*} p<0.05 ;{ }^{* *} p<0.01$. Means, standard deviations, number of biological replicates, and all statistical analyses are reported in Table 1 
(a)

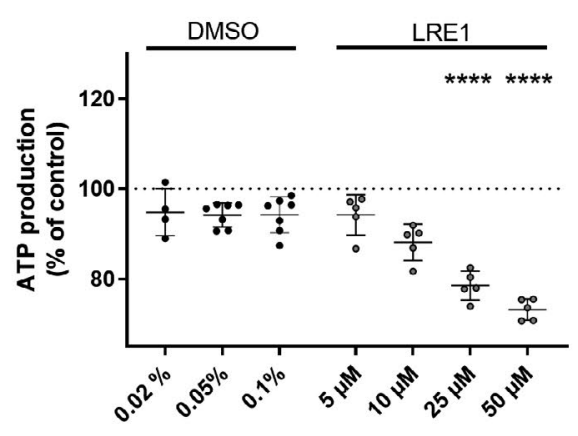

(c)

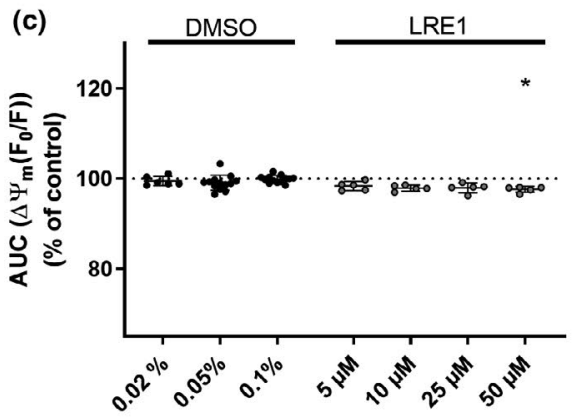

(e)

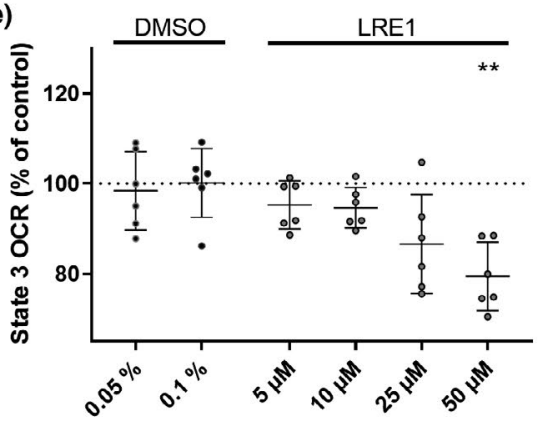

(g)

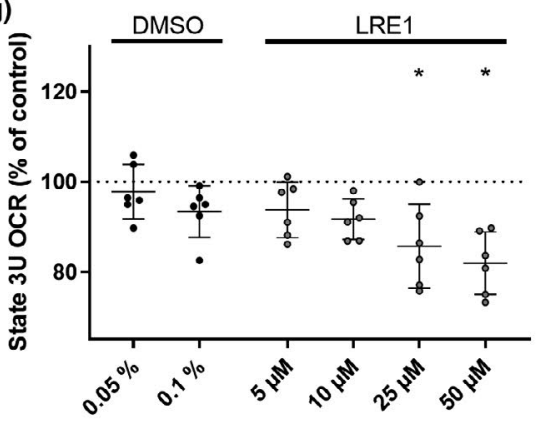

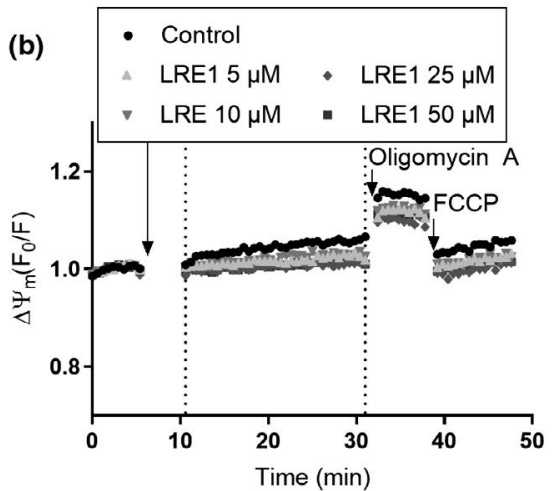
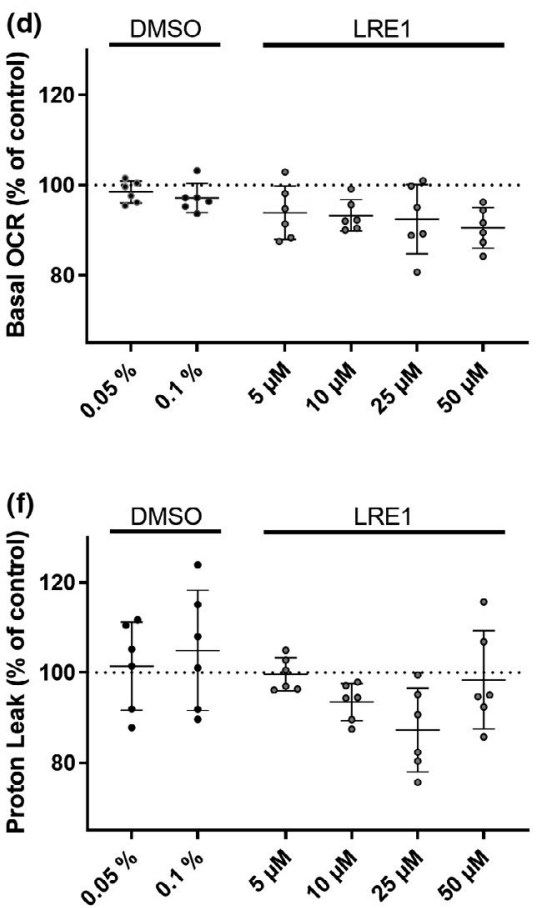

(h)

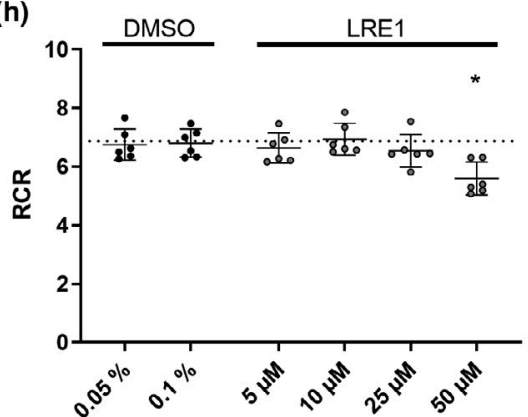

FIGURE 3 Inhibition of sAC decreases mitochondrial respiration and ATP production but have little effect on membrane potential. Oxygen consumption, ATP production, and membrane potential were measured in isolated mitochondria of mouse cerebral cortex and challenged with the newly developed sAC inhibitor LRE1 $(5,10,25$, or $50 \mu \mathrm{M})$. Pyruvate $(10 \mathrm{mM})$ and malate $(2 \mathrm{mM})$ were utilized as substrates and all experiments were performed as described in the Methods section. (a) ATP production normalized to control; $n=5$ for all conditions. (b) Representative trace of membrane potential normalized to baseline measurements (0-5.4 min). Inhibitor or vehicle were added after $5.4 \mathrm{~min}$, oligomycin A were added after $31 \mathrm{~min}$, and FCCP were added after $36 \mathrm{~min}$. (c) AUCs of each trace in panel b were calculated in the interval from 10-31 min (indicated with dotted lines in panel b) normalized to control; $n=5$ for all conditions. (d) Basal respiration normalized to control; $n=6$ for all conditions. (e) State 3 respiration normalized to control; $n=6$ for all conditions. (f) Proton leak normalized to control; $n=6$ for all conditions. (g) State 3 uncoupled respiration; $n=6$ for all conditions. (h) Respiratory control ratio (RCR); $n=6$ for all conditions. Statistics: One-way ANOVA with Dunnett's multiple comparisons test; ${ }^{*} p<0.05 ;{ }^{* *} p<0.01 ;{ }^{* * *} p<0.001$; ${ }^{* * * *} p<0.0001$. Means, standard deviations, number of biological replicates, and all statistical analyses are reported in Tables $2-4$ 
significantly lower than the respective DMSO controls (Figure 5a; Table 2). In terms of membrane potential, CE3F4 (10, 25, or $50 \mu \mathrm{M})$ slightly decreased this to $96 \%-95 \%$ of control, only the latter was significantly lower than the respective DMSO control (Figure $5 b,+C$; Table 2). Further, CE3F4 had no effect on basal OCR (Figure $5 \mathrm{~d}$; Tables 3 and 4). CE3F4 significantly increased state 3 OCR to $127 \%-132 \%$ of control (Figure 5e; Tables 3 and 4), without having a significant effect on proton leak (Figure 5f; Table 3,4). CE3F4 had no effect on state 3 uncoupled OCR (Figure 5g, Tables 3 and 4). CE3F4 of 10, 25, or $50 \mu \mathrm{M}$ significantly increased RCR to 8.2, 8.2, and 7.9, respectively (Figure 5h; Tables 3 and 4). (a)
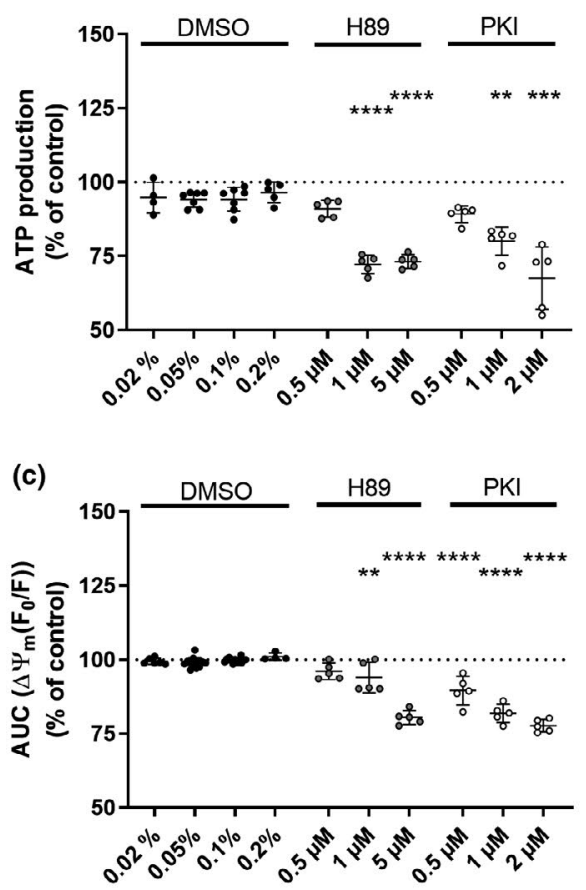

(e)

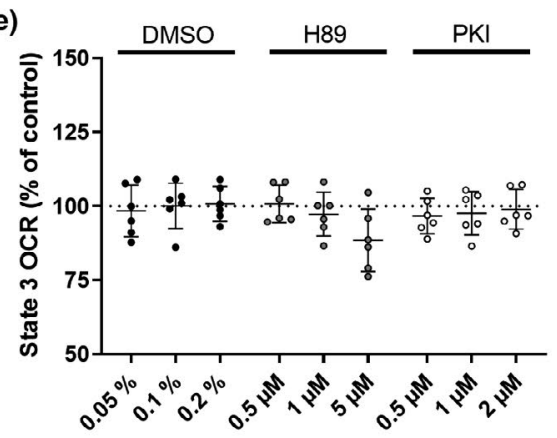

(g)

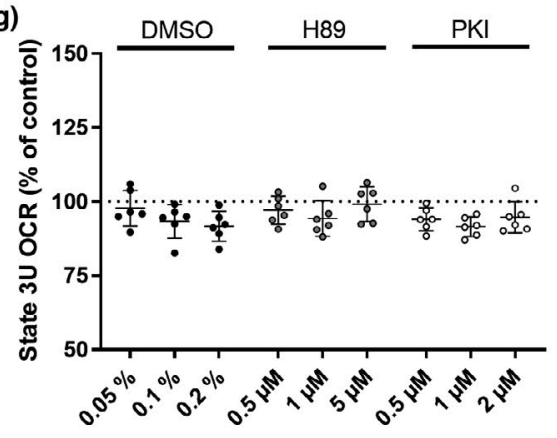

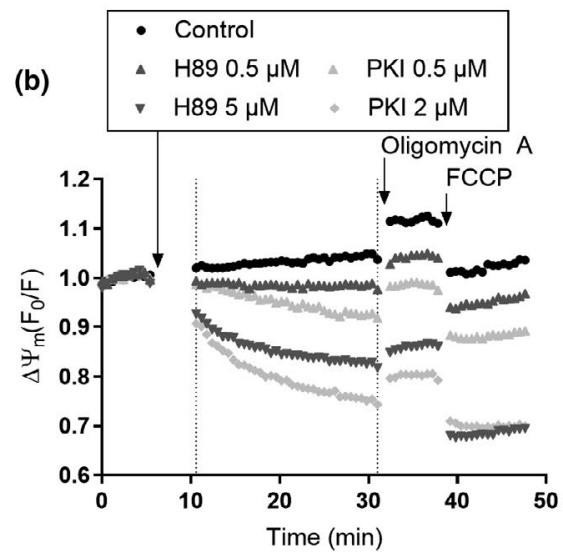
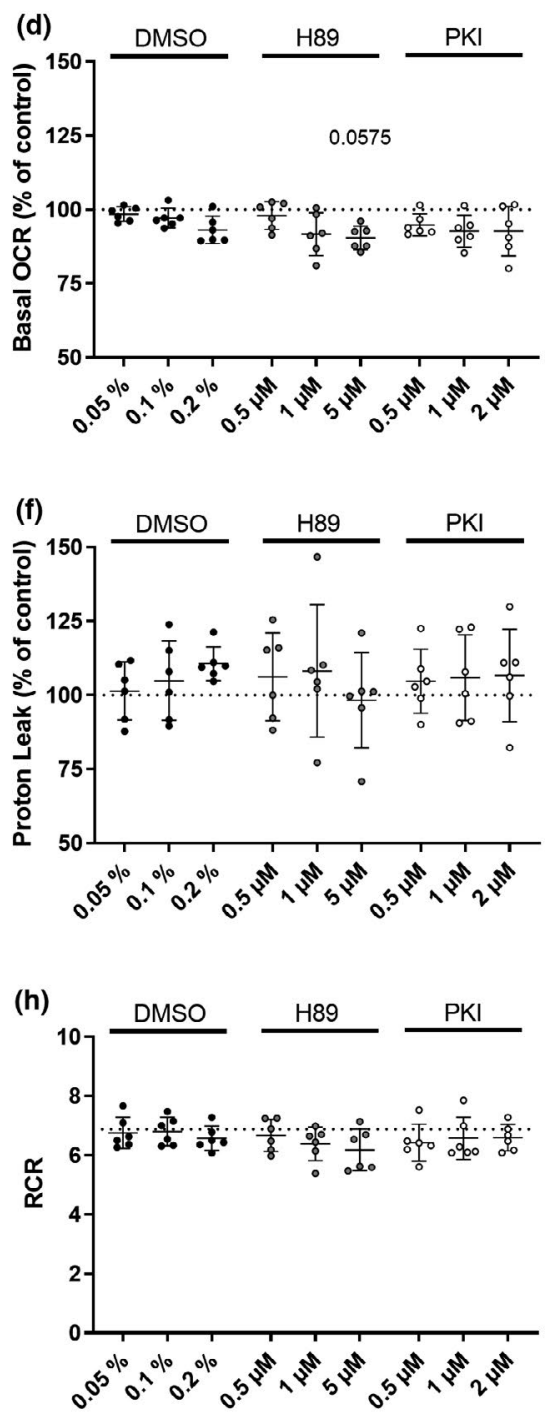
FIGURE 4 Inhibition of PKA decreases ATP production and membrane potential without effecting respiration in isolated mouse cerebral cortex mitochondria. Oxygen consumption, ATP production, and membrane potential were measured in isolated mitochondria of mouse cerebral cortex and challenged with the PKA inhibitors H89 $(0.5,1$, or $5 \mu \mathrm{M})$ or PKI $(0.5$, 1, or $2 \mu \mathrm{M})$. Pyruvate $(10 \mathrm{mM})$ and malate $(2 \mathrm{mM})$ were utilized as substrates and all experiments were performed as described in the Methods section. (a) ATP production normalized to control; $n=5$ for all conditions. (b) Representative trace of membrane potential normalized to baseline measurements (0-5.4 min). Inhibitor or vehicle were added after $5.4 \mathrm{~min}$, oligomycin A were added after $31 \mathrm{~min}$ and FCCP were added after 36 min. (c) AUCs of each trace in panel B were calculated in the interval from 10-31 min (indicated with dotted lines in panel B) normalized to control; $n=5$ for all conditions. (d) Basal respiration normalized to control; $n=6$ for all conditions. (e) State 3 respiration normalized to control; $n=6$ for all conditions. (f) Proton leak normalized to control; $n=6$ for all conditions. (g) State 3 uncoupled respiration; $n=6$ for all conditions. H: Respiratory control ratio (RCR); $n=6$ for all conditions. Statistics: One-way ANOVA with Dunnett's multiple comparisons test, except for $2 \mu M$ PKI which was compared to DMSO $0.2 \%$ with an unpaired $t$ test; ${ }^{*} p<0.05 ;{ }^{* *} p<0.01 ;{ }^{* * *} p<0.001 ;{ }^{* * * *} p<0.0001$. Means, standard deviations, number of biological replicates, and all statistical analyses are reported in Tables $2-4$

\section{4 | DISCUSSION}

To our knowledge, the role of intramitochondrial cAMP in regulating OXPHOS in cerebral mitochondria only includes three publications to this date (Acin-Perez, Russwurm, et al., 2011; Acin-Perez, Salazar, Brosel, et al., 2009; Jakobsen et al., 2018). Acin-Perez and colleagues reported that in mouse brain mitochondria inhibition of $\mathrm{SAC}$ with $\mathrm{KH} 7$ lowers intramitochondrial cAMP levels, ATP production, and respiration (Acin-Perez, Russwurm, et al., 2011; Acin-Perez, Salazar, Brosel, et al., 2009). Furthermore, they reported that the cAMP analog $8 \mathrm{Br}$ cAMP increased ATP production and respiration, while $\mathrm{H} 89$ lowered ATP production and Complex IV activity (Acin-Perez, Russwurm, et al., 2011; Acin-Perez, Salazar, Brosel, et al., 2009). Recently, we reported that inhibition of SAC in isolated cerebral cortical mitochondria lowered ATP production and respiration (Jakobsen et al., 2018).

\section{1 | Effect of DMSO on mitochondrial function}

In order to separate the effect of the pharmacological treatments and the potential effect of DMSO, we assessed the effect of DMSO on mitochondrial function in the concentrations employed as vehicles. DMSO slightly lowered ATP production, while showing no measurable effect on the membrane potential, which could suggest a direct effect of DMSO on ATP synthase as suggested previously (de Meis \& Suzano, 1988). DMSO caused a dose-dependent increase in the proton leak, which triggered a small nonsignificant decrease in RCR which could be explained by DMSO's effect on the permeability of lipid membranes (Notman et al., 2006). State 3 respiration were unchanged while basal and state 3 uncoupled respiration were lowered at higher DMSO concentrations. The latter observations suggest that DMSO has an effect either on the TCA cycle or on the ETC independent of its effect on ATP synthase.

\subsection{Inhibition of SAC decreases OXPHOS in isolated brain cortex mitochondria}

Here, we observe that inhibition of SAC by LRE1 lowers mitochondrial ATP production and both state 3 and state 3 uncoupled respiration in a dose-dependent manner. This support previous reports that mitochondria in the cerebral cortex have an intramitochondrial SAC-cAMP-mediated pathway that regulates OXPHOS (Acin-Perez,
Russwurm, et al., 2011; Acin-Perez, Salazar, Brosel, et al., 2009; Jakobsen et al., 2018). Inhibition of sAC with LRE1 caused a small decrease in membrane potential, a finding that was also reported by Ramos-Espiritu when applying 50 or $100 \mu \mathrm{M}$ LRE1 to mouse brain mitochondria (Ramos-Espiritu et al., 2016). We recently showed that inhibition of $\mathrm{SAC}$ with low concentrations of 2-OHE $(10 \mu \mathrm{M})$ or $\mathrm{KH} 7(1 \mu \mathrm{M})$ had no effect on the membrane potential of isolated cerebral cortex mitochondria despite inducing a decrease in ATP production and respiration (Jakobsen et al., 2018). In contrast, AcinPerez and colleagues showed that inhibition of sAC with $25 \mu \mathrm{M} \mathrm{KH7}$ decreased mitochondrial membrane potential (Acin-Perez, Salazar, Kamenetsky, et al., 2009); however, several more recent studies have revealed that $\mathrm{KH} 7$ works as a mitochondrial uncoupler at concentrations of above $10 \mu \mathrm{M}$ (Di Benedetto et al., 2013; Jakobsen et al., 2018; Ramos-Espiritu et al., 2016). Thus, in line with previous reports from mouse brain mitochondria, we find that inhibition of SAC causes a decrease in OXPHOS in mouse cerebral cortex mitochondria. While these results do not reveal any information regarding the downstream pathway that leads to regulation of OXPHOS, they provide a novel, valuable pharmacological characterization of LRE1's effects on respiration, membrane potential, and ATP production in isolated mouse cerebral cortex mitochondria.

\subsection{Inhibition of PKA decreases ATP production and membrane potential without affecting respiration in isolated mouse cerebral cortex mitochondria}

Inhibiting PKA with the selective inhibitor PKI significantly decreased ATP production and the membrane potential in a very similar manner; however, PKI induced no effect on respiration. When applying $\mathrm{H} 89$, similar responses as those induced by $\mathrm{PKI}$ were observed on ATP production and membrane potential. In line with this, Acin-Perez and colleagues previously reported that $1 \mu \mathrm{M} \mathrm{H} 89$ induced a similar decrease in ATP production in isolated brain mitochondria (Acin-Perez, Russwurm, et al., 2011; Acin-Perez, Salazar, Brosel, et al., 2009). Similar to what was observed with PKI, H89 had no significant effect on respiration; however, a high concentration $(5 \mu \mathrm{M})$ showed a tendency toward decreased basal respiration. A possible explanation for the tendency toward an effect on basal respiration observed when applying a high concentration of $\mathrm{H} 89$, but 
(a)

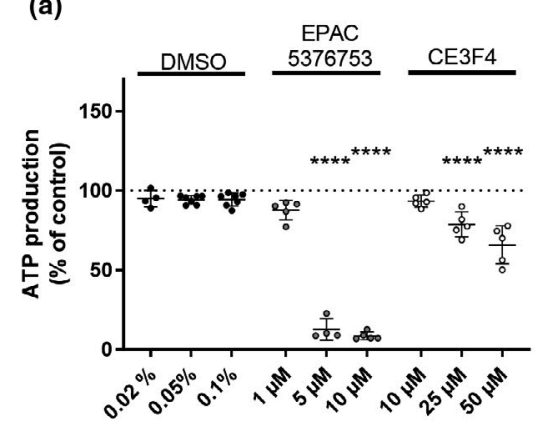

(c)

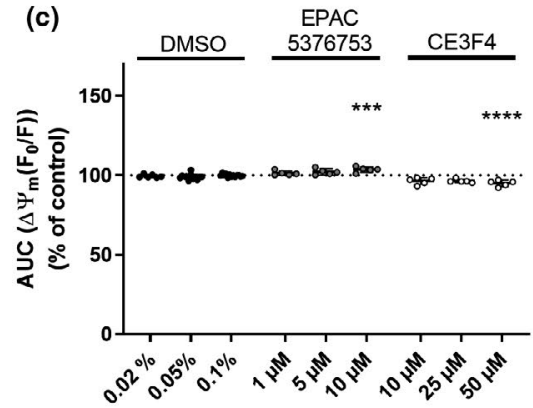

(e)
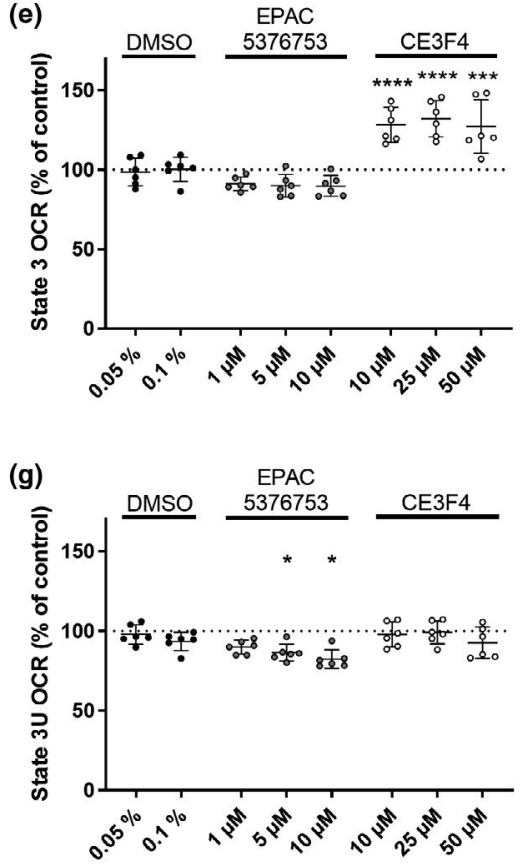
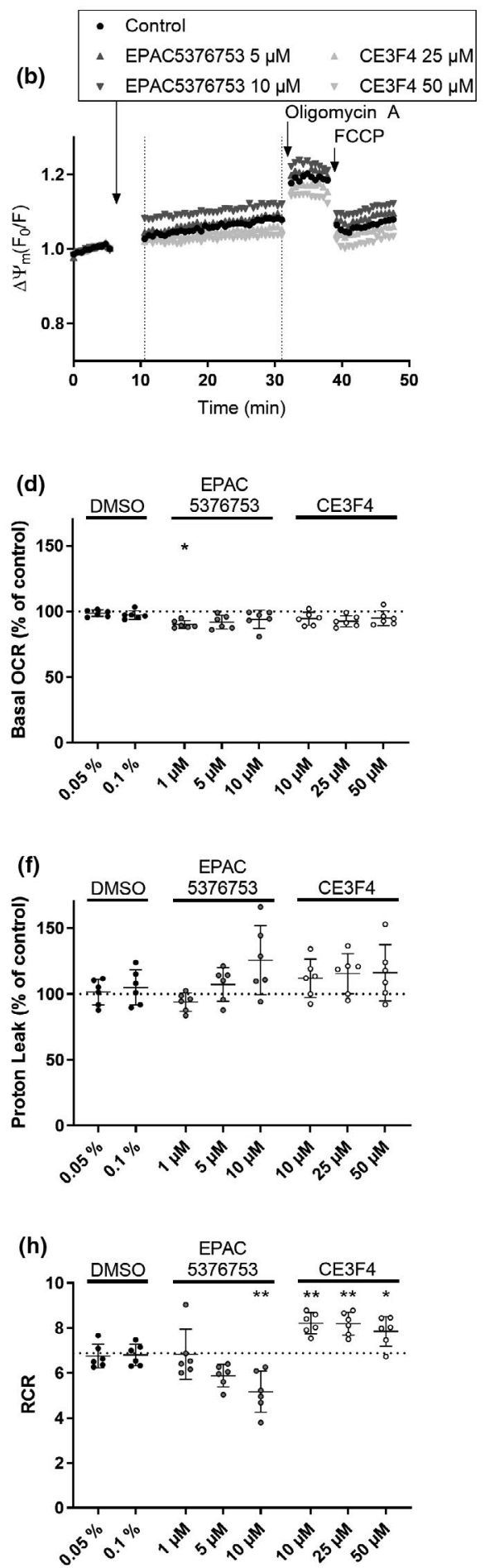

FIGURE 5 Uncompetitive inhibition of EPAC decreases mitochondrial membrane potential leading to downstream effects on ATP production and respiration. Oxygen consumption, ATP production, and membrane potential were measured in isolated mitochondria of mouse cerebral cortex and challenged with the noncompetitive inhibitor of EPAC1; EPAC $5376753(1,5$ or $10 \mu \mathrm{M})$ or the uncompetitive inhibitor CE3F4 $(10,25$, or $50 \mu \mathrm{M})$. Pyruvate $(10 \mathrm{mM})$ and malate $(2 \mathrm{mM})$ were utilized as substrates and all experiments were performed as described in the Methods section. (a) ATP production normalized to control; $n=5$ for all conditions, except EPAC $53767535 \mu M$ with $n=4$. (b) Representative trace of membrane potential normalized to baseline measurements (0-5.4 min). Inhibitor or vehicle were added after $5.4 \mathrm{~min}$, oligomycin A were added after $31 \mathrm{~min}$ and FCCP were added after $36 \mathrm{~min}$. (c) AUCs of each trace in panel b were calculated in the interval from 10-31 min (indicated with dotted lines in panel b) normalized to control; $n=5$ for all conditions. (d) Basal respiration normalized to control; $n=6$ for all conditions. (e) State 3 respiration normalized to control; $n=6$ for all conditions. (f) Proton leak normalized to control; $n=6$ for all conditions. (g) State 3 uncoupled respiration; $n=6$ for all conditions. (h) Respiratory control ratio (RCR); $n=6$ for all conditions. Statistics: One-way ANOVA with Dunnett's multiple comparisons test; ${ }^{*} p<0.05 ;{ }^{* *} p<0.01 ;{ }^{* * *} p<0.001 ;{ }^{* * *} p<0.0001$. Means, standard deviations, number of biological replicates, and all statistical analyses are reported in Tables 2-4 
not PKI, could be a non-PKA-mediated effect of $\mathrm{H} 89 . \mathrm{H} 89$ has been suggested to inhibit at least five other protein kinases with IC50 values at or below 2.8 MM (Davies, Reddy, Caivano, \& Cohen, 2000). In the presence of either inhibitor an effect on ATP production and membrane potential were observed at concentrations that had no effect on state 3 respiration. This observation suggests that PKA in cerebral cortical mitochondria can regulate the capacity for ATP production potentially by disrupting the membrane potential (and thereby lowering the potential for ATP synthesis) independent of changes in respiration. PKA-mediated phosphorylation of BCL-2 antagonist of cell death (BAD) is protecting against mitochondrial outer membrane permeability by allowing $B A D$ to bind to and thereby inhibit BCL-2 (Chipuk \& Green, 2008; Lizcano, Morrice, \& Cohen, 2000). The decrease in membrane potential observed when inhibiting PKA, which is independent of changes in OCR, suggest that the permeability of the OMM is regulated by PKA activity. This could potentially be mediated via the BAD signaling pathway, although this cannot be concluded based on the results presented in this study.

Besides the risk of off-target effects, pharmacological inhibition of PKA has the pitfall that the inhibitors do not distinguish between PKA in the matrix, the IMS, or OMM. Hence, the results presented here cannot be employed to conclude that the involved PKA phosphorylation sites are found in the matrix. However, as discussed above, these results do not support that a PKA phosphorylation target is able to regulate ETC activity and thereby regulating OXPHOS in brain mitochondria as has been suggested for Ser58 on the matrix side of Complex IV-1 in mitochondria from other cells and tissue (Acin-Perez, Gatti, et al., 2011).

\subsection{Uncompetitive inhibition of EPAC decreases mitochondrial membrane potential possibly leading to downstream effects on ATP production and respiration}

EPAC1 in mitochondria has gained increased attention recently and has been suggested to play a role in regulating MCU activity in H295R adrenocortical cells and HeLa cells as well as MCU activity and OXPHOS activity in isolated cardiac mitochondria from mouse (Szanda, Wisniewski, Rajki, \& Spät, 2018; Wang et al., 2016). EPAC1 has not yet been demonstrated in brain mitochondria (to our knowledge), but here we provide pharmacological evidence for its functional presence.

To assess the effect of EPAC1 in cerebral cortical mitochondria, we apply two different inhibitors of EPAC1, the uncompetitive inhibitor CE3F4 and the noncompetitive inhibitor EPAC 5376753. We observe no effect of CE3F4 on basal respiration. However, this may be expected since the effect of an uncompetitive inhibitor downstream of cAMP may be questionable during baseline respiration since the intramitochondrial CAMP levels are expected to be lower due to the respiratory control and thereby decreased TCA cycle activity (which compared to state 3 respiration leads to less production of $\mathrm{CO}_{2} \rightarrow$ less bicarbonate $\rightarrow$ less SAC activation). When state 3 is induced with the presence of ADP (increased TCA cycle activity $\rightarrow$ increased SAC activity $\rightarrow$ increased concentration of CAMP and thereby inhibition of EPAC1 by CE3F4 must be expected), we observe a small decrease in membrane potential, which could explain the observed decrease in ATP synthesis as well increased state 3 respiration to compensate for the decreased membrane potential. This could indicate that EPAC1 is involved in regulating the membrane potential which would also explain why no effect of CE3F4 is observed during state 3 uncoupled respiration. These observations suggest an uncoupling effect of CE3F4 which fulfill two of the three general traits of a mitochondrial uncoupler as listed by Terada (1990). However, the lacking increase in baseline respiration and proton leak strongly indicates that CE3F4 does not function as a molecular mitochondrial uncoupler by itself. Wang et al. suggest that EPAC1 has an inhibitory effect on MCU in isolated cardiac mitochondria, and that this mechanism is involved in protecting the mitochondria against MPT (Wang et al., 2016). Our experiments are performed in the absence of $\mathrm{Ca}^{2+}$ making $\mathrm{MCU}$ an unlikely explanation for the observed results; however, the data suggest that EPAC1 is involved in regulating the mitochondrial membrane potential, potentially via other IMM proton transporters such as uncoupling proteins or the adenine nucleotide translocase (Brand et al., 2004).

When applying the noncompetitive inhibitor EPAC 5376753, we observed quite different effects compared to what was observed with CE3F4. Most strikingly EPAC 5376753 inhibited ATP production to the same extent as the inhibitor of ATP synthase oligomycin (oligomycin data not shown). This decreased ATP production was not similar to the effect observed with LRE1 which suggests three possible explanations; (i) EPAC 5376753 has a direct effect on the ATP synthase; (ii) EPAC1 has a baseline activity in the absence of cAMP which can be inhibited to affect ATP synthase; or (iii) EPAC 5376753 interferes directly with the proton gradient. The latter seem unlikely when observing the effect of EPAC 5376753 on membrane potential and respiration, respectively. A direct effect of EPAC 5376753 on ATP synthase could explain the observed increase in membrane potential as well as the decreased basal and state 3 respiration, but does not explain the lowered state 3 uncoupled respiration. The seemingly different effects of CE3F4 and EPAC 5376753 suggest that either one or both have off-target effects not related to the effect of EPAC1, which makes the effect of both inhibitors difficult to interpret and thus caution should be exercised when employing these inhibitors.

\section{5 | Future directions}

Mitochondrial diversity is reported between species, tissues, and even within different regions of the brain (Andersen et al., 2019; Johnson, Harris, French, et al., 2007; Phillips et al., 2012). Due to this diversity, findings in mitochondria from one tissue should only be extrapolated to mitochondria in other tissues if there is experimental data to support it. Based on the current literature and our data, we suggest that PKA plays a role in regulating OXPHOS in cerebral cortical mitochondria. EPAC1 has not been studied extensively in isolated mitochondria; however, its suggested effect on $\mathrm{MCU}$ combined with our results leads to the suggestion that 
EPAC1 also plays a role in regulating OXPHOS in cerebral cortical mitochondria. While we suggest the relevance of both PKA and EPAC1, further studies are needed to verify the downstream targets as well as to elaborate on understanding the pathways mechanistically.

Intramitochondrial cAMP signaling and its downstream effects have been studied in either acutely isolated mitochondria or cell lines. Working in cell lines provides the advantage of being able to express sensors or doing knock out/knock down or overexpression of proteins of interest (e.g., sAC KO cells and HeLa cells expressing a mitochondrial cAMP sensor (Di Benedetto et al., 2013; Valsecchi et al., 2017)). However, one concern when investigating mitochondria is that cell lines rely more heavily on glycolytic metabolism (the Warburg effect and/or the Crabtree effect), and thus their mitochondrial regulation must be expected to differ compared to what is occurring in the brain and other organs highly depending on oxidative metabolism (Diaz-Ruiz, Rigoulet, \& Devin, 2011; Phillips et al., 2012; Semenza, 2000). Thus, working in acutely isolated mitochondria may provide a more accurate insight to the function of in situ brain mitochondria with in vivo transfection prior to the isolation procedure making it possible to manipulate specific proteins of interest. Investigating the mitochondria in primary cultures could for some studies be a useful intermediate model system that quite easily (compared to in vivo) allows for induced expression or knock out/knock down of genes while having mitochondria from cells not affected by the Warburg-Crabtree effect (Kristián, Hopkins, McKenna, \& Fiskum, 2006).

The enzymatic activity of $\mathrm{SAC}$ is increased by $\mathrm{Ca}^{2+}$ while intramitochondrial cAMP seems to affect $\mathrm{Ca}^{2+}$ levels via EPAC1 and subsequently MCU (Steegborn, 2014; Wang et al., 2016). In line with this, it has been reported that rises in mitochondrial $\mathrm{Ca}^{2+}$ increases mitochondrial cAMP (Di Benedetto et al., 2013). Due to this $\mathrm{Ca}^{2+}$ cAMP crosstalk, future studies should address both messengers when wishing to investigate the dynamics of either one.

\section{5 | CONCLUSION}

Here, we have provided a discussion of the current literature on sAC-CAMP-PKA/EPAC signaling in mitochondria and attempted to relate this to brain mitochondria. The literature on this issue is, however, mostly based on experiments in mitochondria from liver or from different non-brain cell lines. To underline the potential importance of this signaling chain in the mammalian brain, we support previous observations of how intramitochondrial SAC regulates OXPHOS in the brain. Furthermore, we provide preliminary evidence of how PKA affects mitochondrial membrane potential and ATP production, seemingly independent of changes in ETC activity. Lastly, we suggest that EPAC1 is functionally present in brain mitochondria where it might regulate membrane potential and thus the capacity for ATP synthesis. Based on this and the evidence that mitochondrial malfunction is a hallmark of numerous neurological diseases, we strongly encourage further research into this important signaling-metabolism coupling in brain mitochondria.

\section{DECLARATION OF TRANSPARENCY}

The authors, reviewers, and editors affirm that in accordance to the policies set by the Journal of Neuroscience Research, this manuscript presents an accurate and transparent account of the study being reported and that all critical details describing the methods and results are present.

\section{ACKNOWLEDGMENTS}

The authors would like to thank the Lundbeck Foundation (EJ; grant no. R249-2017-109), the Augustinus Foundation (LKB), and the Hørslev Foundation (LKB) for providing financial support. Mrs. Janet Nagayi Nandawula da Silva is cordially acknowledged for providing technical support.

\section{CONFLICT OF INTEREST}

The authors declare no potential conflict of interests.

\section{AUTHOR CONTRIBUTIONS}

Conceptualization, EJ, SCL, LKB; Methodology, EJ, SCL, LKB; Investigation, EJ; Formal analysis, EJ; Writing original draft, EJ, LKB; Writing-Review and editing, EJ, SCL, LKB; Visualization, EJ; Supervision, LKB; Funding acquisition, EJ, LKB.

\section{ORCID}

Emil Jakobsen (iD https://orcid.org/0000-0001-6290-4222

Lasse K. Bak iD https://orcid.org/0000-0003-0784-7864

\section{REFERENCES}

Acin-Perez, R., Gatti, D. L., Bai, Y., \& Manfredi, G. (2011). Protein phosphorylation and prevention of cytochrome oxidase inhibition by ATP: Coupled mechanisms of energy metabolism regulation. Cell Metabolism, 13(6), 712-719. https://doi.org/10.1016/j. cmet.2011.03.024

Acin-Perez, R., Russwurm, M., Günnewig, K., Gertz, M., Zoidl, G., Ramos, L., ... Steegborn, C. (2011). A phosphodiesterase 2A isoform localized to mitochondria regulates respiration. Journal of Biological Chemistry, M111, 266379. https://doi.org/10.1074/jbc. M111.266379

Acin-Perez, R., Salazar, E., Brosel, S., Yang, H., Schon, E. A., \& Manfredi, G. (2009). Modulation of mitochondrial protein phosphorylation by soluble adenylyl cyclase ameliorates cytochrome oxidase defects. EMBO Molecular Medicine, 1(8-9), 392-406. https://doi.org/10.1002/ emmm.200900046

Acin-Perez, R., Salazar, E., Kamenetsky, M., Buck, J., Levin, L. R., \& Manfredi, G. (2009). Cyclic AMP produced inside mitochondria 
regulates oxidative phosphorylation. Cell Metabolism, 9(3), 265-276. https://doi.org/10.1016/j.cmet.2009.01.012

Agnes, R. S., Jernigan, F., Shell, J. R., Sharma, V., \& Lawrence, D. S. (2010). Suborganelle sensing of mitochondrial CAMP-dependent protein kinase activity. Journal of the American Chemical Society, 132(17), 60756080. https://doi.org/10.1021/ja909652q

Andersen, J. V., Christensen, S. K., Nissen, J. D., \& Waagepetersen, H. S. (2017). Improved cerebral energetics and ketone body metabolism in db/db mice. Journal of Cerebral Blood Flow \& Metabolism, 37(3), 1137-1147. https://doi.org/10.1177/0271678X16684154

Andersen, J. V., Jakobsen, E., Waagepetersen, H. S., \& Aldana, B. I. (2019). Distinct differences in rates of oxygen consumption and ATP synthesis of regionally isolated non-synaptic mouse brain mitochondria. Journal of Neuroscience Research, 2019, 1-14. https://doi.org/10.1002/jnr.24371

Brand, M. D., Affourtit, C., Esteves, T. C., Green, K., Lambert, A. J., Miwa, S., ... Parker, N. (2004). Mitochondrial superoxide: Production, biological effects, and activation of uncoupling proteins. Free Radical Biology and Medicine, 37(6), 755-767. https://doi.org/10.1016/j.freeradbiomed.2004.05.034

Brescia, M., \& Zaccolo, M. (2016). Modulation of compartmentalised cyclic nucleotide signalling via local inhibition of phosphodiesterase activity. International Journal of Molecular Sciences, 17(10), 1672. https ://doi.org/10.3390/ijms17101672

Brown, L. M., Rogers, K. E., Aroonsakool, N., McCammon, J. A., \& Insel, P. A. (2014). Allosteric inhibition of Epac: Computational modeling and experimental validation to identify allosteric sites and inhibitors. Journal of Biological Chemistry, M114, 569319. https://doi.org/10.1074/jbc. M114.569319

Buck, J., \& Levin, L. (2011). Physiological sensing of carbon dioxide/bicarbonate/pH via cyclic nucleotide signaling. Sensors, 11(2), 2112. https ://doi.org/10.3390/s110202112

Burdyga, A., Surdo, N. C., Monterisi, S., Di Benedetto, G., Grisan, F., Penna, E., ... Lefkimmiatis, K. (2018). Phosphatases control PKAdependent functional microdomains at the outer mitochondrial membrane. Proceedings of the National Academy of Sciences, 115(28), E6497-E6506. https://doi.org/10.1073/pnas.1806318115

Buxton, I., \& Brunton, L. (1983). Compartments of cyclic AMP and protein kinase in mammalian cardiomyocytes. Journal of Biological Chemistry, 258(17), 10233-10239.

Chen, Y., Cann, M. J., Litvin, T. N., lourgenko, V., Sinclair, M. L., Levin, L. R., \& Buck, J. (2000). Soluble adenylyl cyclase as an evolutionarily conserved bicarbonate sensor. Science, 289(5479), 625-628.

Cheng, X., Ji, Z., Tsalkova, T., \& Mei, F. (2008). Epac and PKA: A tale of two intracellular cAMP receptors. Acta Biochimica et Biophysica Sinica, 40(7), 651-662. https://doi.org/10.1111/j.1745-7270.2008.00438.x

Chipuk, J. E., \& Green, D. R. (2008). How do BCL-2 proteins induce mitochondrial outer membrane permeabilization? Trends in Cell Biology, 18(4), 157-164. https://doi.org/10.1016/j.tcb.2008.01.007

Conover, T. E. (1969). Influence of organic solutes on the reactions of oxidative phosphorylation. Journal of Biological Chemistry, 244(2), 254-259.

Cooper, D. M. (2005). Compartmentalization of adenylate cyclase and cAMP signalling. Biochemical Society Transactions, 33(Pt 6), 13191322. https://doi.org/10.1042/bst20051319

Cooper, D. M., \& Tabbasum, V. G. (2014). Adenylate cyclase-centred microdomains. Biochemical Journal, 462(2), 199-213. https://doi. org/10.1042/BJ20140560

Correia, S. C., Santos, R. X., Perry, G., Zhu, X., Moreira, P. I., \& Smith, M. A. (2012). Mitochondrial importance in Alzheimer's, Huntington's and Parkinson's diseases. In S. I. Ahmad (Ed.), Neurodegenerative diseases (pp. 205-221). New York, NY: Springer, US.

Courilleau, D., Bisserier, M., Jullian, J.-C., Lucas, A., Bouyssou, P., Fischmeister, R., ... Lezoualc'h, F. (2012). Identification of a tetrahydroquinoline analog as a pharmacological inhibitor of the CAMPbinding protein Epac. Journal of Biological Chemistry, M112, 422956. https://doi.org/10.1074/jbc.M112.422956
Covian, R., French, S., Kusnetz, H., \& Balaban, R. S. (2014). Stimulation of oxidative phosphorylation by calcium in cardiac mitochondria is not influenced by cAMP and PKA activity. Biochimica et Biophysica Acta Bioenergetics, 1837(12), 1913-1921.

Dalton, G. D., \& Dewey, W. L. (2006). Protein kinase inhibitor peptide (PKI): A family of endogenous neuropeptides that modulate neuronal cAMP-dependent protein kinase function. Neuropeptides, 40(1), 23-34. https://doi.org/10.1016/j.npep.2005.10.002

Davies, S. P., Reddy, H., Caivano, M., \& Cohen, P. (2000). Specificity and mechanism of action of some commonly used protein kinase inhibitors. The Biochemical Journal, 351(Pt 1), 95-105. https://doi. org/10.1042/bj3510095

de Meis, L., \& Suzano, V. A. (1988). Role of water activity on the rates of acetyl phosphate and ATP hydrolysis. FEBS Letters, 232(1), 73-77. https://doi.org/10.1016/0014-5793(88)80389-2

Dessauer, C. W. (2009). Adenylyl cyclise-A-kinase anchoring protein complexes: The next dimension in cAMP signaling. Molecular Pharmacology, 76(5), 935-941. https://doi.org/10.1124/mol.109.059345

Di Benedetto, G., Gerbino, A., \& Lefkimmiatis, K. (2018). Shaping mitochondrial dynamics: The role of CAMP signalling. Biochemical and Biophysical Research Communications, 500(1), 65-74. https://doi. org/10.1016/j.bbrc.2017.05.041

Di Benedetto, G., Scalzotto, E., Mongillo, M., \& Pozzan, T. (2013). Mitochondrial $\mathrm{Ca} 2+$ uptake induces cyclic AMP generation in the matrix and modulates organelle ATP levels. Cell Metabolism, 17(6), 965-975. https://doi.org/10.1016/j.cmet.2013.05.003

Diaz-Ruiz, R., Rigoulet, M., \& Devin, A. (2011). The Warburg and Crabtree effects: On the origin of cancer cell energy metabolism and of yeast glucose repression. Biochimica et Biophysica Acta - Bioenergetics, 1807(6), 568-576.

Dodge-Kafka, K. L., Soughayer, J., Pare, G. C., Michel, J. J. C., Langeberg, L. K., Kapiloff, M. S., \& Scott, J. D. (2005). The protein kinase A anchoring protein mAKAP coordinates two integrated cAMP effector pathways. Nature, 437(7058), 574. https://doi.org/10.1038/natur e03966

Gaignard, P., Liere, P., Thérond, P., Schumacher, M., Slama, A., \& Guennoun, R. (2017). Role of sex hormones on brain mitochondrial function, with special reference to aging and neurodegenerative diseases. Frontiers in Aging Neuroscience, 9, 406. https://doi. org/10.3389/fnagi.2017.00406

Grimsrud, P. A., Carson, J. J., Hebert, A. S., Hubler, S. L., Niemi, N. M., Bailey, D. J., ... Pagliarini, D. J. (2012). A quantitative map of the liver mitochondrial phosphoproteome reveals posttranslational control of ketogenesis. Cell Metabolism, 16(5), 672-683. https://doi. org/10.1016/j.cmet.2012.10.004

Harris, J. J., Jolivet, R., \& Attwell, D. (2012). Synaptic energy use and supply. Neuron, 75(5), 762-777. https://doi.org/10.1016/j. neuron.2012.08.019

Horvat, A., \& Vardjan, N. (2018). Astroglial cAMP signalling in space and time. Neuroscience Letters, 689, 5-10. https://doi.org/10.1016/j. neulet.2018.06.025

Howarth, C., Gleeson, P., \& Attwell, D. (2012). Updated energy budgets for neural computation in the neocortex and cerebellum. Journal of Cerebral Blood Flow \& Metabolism, 32(7), 1222-1232. https://doi. org/10.1038/jcbfm.2012.35

Jakobsen, E., Lange, S. C., Andersen, J. V., Desler, C., Kihl, H., Hohnholt, M. C., Bak, L. K. (2018, September 1). The inhibitors of soluble adenylate cyclase 2-OHE, $\mathrm{KH} 7$, and bithionol compromise mitochondrial ATP production by distinct mechanisms. Biochemical Pharmacology, 155, 92-101.

Johnson, D. T., Harris, R. A., Blair, P. V., \& Balaban, R. S. (2007). Functional consequences of mitochondrial proteome heterogeneity. American Journal of Physiology-Cell Physiology, 292(2), C698-C707. https://doi. org/10.1152/ajpcell.00109.2006

Johnson, D. T., Harris, R. A., French, S., Blair, P. V., You, J., Bemis, K. G., ... Balaban, R. S. (2007). Tissue heterogeneity of the 
mammalian mitochondrial proteome. American Journal of PhysiologyCell Physiology, 292(2), C689-C697. https://doi.org/10.1152/ajpce II.00108.2006

Kokkonen, K., \& Kass, D. A. (2017). Nanodomain regulation of cardiac cyclic nucleotide signaling by phosphodiesterases. Annual Review of Pharmacology and Toxicology, 57, 455-479. https://doi.org/10.1146/ annurev-pharmtox-010716-104756

Kristián, T., Hopkins, I. B., McKenna, M. C., \& Fiskum, G. (2006). Isolation of mitochondria with high respiratory control from primary cultures of neurons and astrocytes using nitrogen cavitation. Journal of Neuroscience Methods, 152(1-2), 136-143. https://doi.org/10.1016/j. jneumeth.2005.08.018

Kritzer, M. D., Li, J., Dodge-Kafka, K., \& Kapiloff, M. S. (2012). AKAPs: The architectural underpinnings of local cAMP signaling. Journal of Molecular and Cellular Cardiology, 52(2), 351-358. https://doi. org/10.1016/j.yjmcc.2011.05.002

Lanza, I. R., \& Nair, K. S. (2009). Functional assessment of isolated mitochondria in vitro. Methods in Enzymology, 457, 349-372. https://doi. org/10.1016/S0076-6879(09)05020-4

Lefkimmiatis, K., Leronni, D., \& Hofer, A. M. (2013). The inner and outer compartments of mitochondria are sites of distinct CAMP/PKA signaling dynamics. Journal of Cell Biology, 202(3), 453-462. https://doi. org/10.1083/jcb.201303159

Lefkimmiatis, K., \& Zaccolo, M. (2014). cAMP signaling in subcellular compartments. Pharmacology \& Therapeutics, 143(3), 295-304. https ://doi.org/10.1016/j.pharmthera.2014.03.008

Litvin, T. N., Kamenetsky, M., Zarifyan, A., Buck, J., \& Levin, L. R. (2003). Kinetic properties of "soluble" adenylyl cyclase synergism between calcium and bicarbonate. Journal of Biological Chemistry, 278(18), 15922-15926. https://doi.org/10.1074/jbc.M212475200

Lizcano, J. M., Morrice, N., \& Cohen, P. (2000). Regulation of BAD by cAMP-dependent protein kinase is mediated via phosphorylation of a novel site, Ser155. Biochemical Journal, 349(2), 547-557. https:// doi.org/10.1042/bj3490547

Lochner, A., \& Moolman, J. (2006). The many faces of H89: A review. Cardiovascular Drug Reviews, 24(3-4), 261-274. https://doi. org/10.1111/j.1527-3466.2006.00261.x

Manfredi, G., Yang, L., Gajewski, C. D., \& Mattiazzi, M. (2002). Measurements of ATP in mammalian cells. Methods, 26(4), 317-326. https://doi.org/10.1016/S1046-2023(02)00037-3

Mink, J. W., Blumenschine, R. J., \& Adams, D. B. (1981). Ratio of central nervous system to body metabolism in vertebrates: Its constancy and functional basis. American Journal of Physiology-Regulatory, Integrative and Comparative Physiology, 241(3), R203-R212. https:// doi.org/10.1152/ajpregu.1981.241.3.R203

Monterisi, S., Lobo, M. J., Livie, C., Castle, J. C., Weinberger, M., Baillie, G., ... Zaccolo, M. (2017). PDE2A2 regulates mitochondria morphology and apoptotic cell death via local modulation of CAMP/PKA signalling. Elife, 6, e21374. https://doi.org/10.7554/eLife.21374

Monterisi, S., \& Zaccolo, M. (2017). Components of the mitochondrial cAMP signalosome. Biochemical Society Transactions, 45(1), 269-274. https://doi.org/10.1042/BST20160394

Nasrallah, F. A., Garner, B., Ball, G. E., \& Rae, C. (2008). Modulation of brain metabolism by very low concentrations of the commonly used drug delivery vehicle dimethyl sulfoxide (DMSO). Journal of Neuroscience Research, 86(1), 208-214. https://doi.org/10.1002/ jnr.21477

Nicholls, D. G., \& Ward, M. W. (2000). Mitochondrial membrane potential and neuronal glutamate excitotoxicity: Mortality and millivolts. Trends in Neurosciences, 23(4), 166-174. https://doi.org/10.1016/ S0166-2236(99)01534-9

Notman, R., Noro, M., O'Malley, B., \& Anwar, J. (2006). Molecular basis for dimethylsulfoxide (DMSO) action on lipid membranes. Journal of the American Chemical Society, 128(43), 13982-13983. https://doi. org/10.1021/ja063363t
Phillips, D., Covian, R., Aponte, A. M., Glancy, B., Taylor, J. F., Chess, D., \& Balaban, R. S. (2012). Regulation of oxidative phosphorylation complex activity: Effects of tissue-specific metabolic stress within an allometric series and acute changes in workload. American Journal of Physiology-Regulatory, Integrative and Comparative Physiology, 302(9), R1034-R1048. https://doi.org/10.1152/ajpregu.00596.2011

Qiao, J., Mei, F. C., Popov, V. L., Vergara, L. A., \& Cheng, X. (2002). Cell cycle dependent subcellular localization of exchange factor directly activated by CAMP. Journal of Biological Chemistry, 277(29), 2658126586. https://doi.org/10.1074/jbc.M203571200

Ramos-Espiritu, L., Kleinboelting, S., Navarrete, F. A., Alvau, A., Visconti, P. E., Valsecchi, F., ... Buck, J. (2016). Discovery of LRE1 as a specific and allosteric inhibitor of soluble adenylyl cyclase. Nature Chemical Biology, 12(10), 838. https://doi.org/10.1038/nchembio.2151

Schmidt, M., Dekker, F. J., \& Maarsingh, H. (2013). Exchange protein directly activated by cAMP (epac): A multidomain cAMP mediator in the regulation of diverse biological functions. Pharmacological Reviews, 65(2), 670-709. https://doi.org/10.1124/pr.110.003707

Scott, J. D., Dessauer, C. W., \& Taskén, K. (2013). Creating order from chaos: Cellular regulation by kinase anchoring. Annual Review of Pharmacology and Toxicology, 53, 187-210. https://doi.org/10.1146/ annurev-pharmtox-011112-140204

Semenza, G. L. (2000). Hypoxia, clonal selection, and the role of HIF-1 in tumor progression. Critical Reviews in Biochemistry and Molecular Biology, 35(2), 71-103. https://doi.org/10.1080/1040923009 1169186

Steegborn, C. (2014). Structure, mechanism, and regulation of soluble adenylyl cyclases-Similarities and differences to transmembrane adenylyl cyclases. Biochimica et Biophysica Acta - Molecular Basis of Disease, 1842(12. Part B), 2535-2547. https://doi.org/10.1016/j. bbadis.2014.08.012

Steegborn, C., Litvin, T. N., Hess, K. C., Capper, A. B., Taussig, R., Buck, J., ... Wu, H. (2005). A novel mechanism for adenylyl cyclase inhibition from the crystal structure of its complex with catechol estrogen. Journal of Biological Chemistry, 280(36), 31754-31759. https://doi. org/10.1074/jbc.M507144200

Steegborn, C., Litvin, T. N., Levin, L. R., Buck, J., \& Wu, H. (2005). Bicarbonate activation of adenylyl cyclase via promotion of cataIytic active site closure and metal recruitment. Nature Structural \& Molecular Biology, 12(1), 32-37. https://doi.org/10.1038/nsmb880

Szanda, G., Wisniewski, É., Rajki, A., \& Spät, A. (2018). Mitochondrial cAMP exerts positive feedback on mitochondrial $\mathrm{Ca} 2+$ uptake via the recruitment of Epac1. Journal of Cell Science, 131(10), jcs215178.

Terada, H. (1990). Uncouplers of oxidative phosphorylation. Environmental Health Perspectives, 87, 213. https://doi.org/10.1289/ehp.9087213

Theurkauf, W. E., \& Vallee, R. (1982). Molecular characterization of the cAMP-dependent protein kinase bound to microtubule-associated protein 2. Journal of Biological Chemistry, 257(6), 3284-3290.

Valsecchi, F., Konrad, C., D'Aurelio, M., Ramos-Espiritu, L. S., Stepanova, A., Burstein, S. R., ... Buck, J. (2017). Distinct intracellular sAC-cAMP domains regulate ER calcium signaling and OXPHOS function. Journal of Cell Science, 130, 3713-3727.

Valsecchi, F., Konrad, C., \& Manfredi, G. (2014). Role of soluble adenyIyl cyclase in mitochondria. Biochimica Et Biophysica Acta - Molecular Basis of. Disease, 1842(12), 2555-2560. https://doi.org/10.1016/j. bbadis.2014.05.035

Valsecchi, F., Ramos-Espiritu, L. S., Buck, J., Levin, L. R., \& Manfredi, G. (2013). cAMP and mitochondria. Physiology, 28(3), 199-209. https:// doi.org/10.1152/physiol.00004.2013

Wang, Z., Liu, D., Varin, A., Nicolas, V., Courilleau, D., Mateo, P., ... Brenner, C. (2016). A cardiac mitochondrial cAMP signaling pathway regulates calcium accumulation, permeability transition and cell death. Cell Death \& Disease, 7(4), e2198. https://doi.org/10.1038/cddis.2016.106

Wiggins, S. V., Steegborn, C., Levin, L. R., \& Buck, J. (2018). Pharmacological modulation of the $\mathrm{CO} 2 / \mathrm{HCO}^{-} / \mathrm{pH}-$, calcium-, and 
ATP-sensing soluble adenylyl cyclase. Pharmacology \& Therapeutics, 190, 173-186. https://doi.org/10.1016/j.pharmthera.2018.05.008

Zhang, F., Qi, Y., Zhou, K., Zhang, G., Linask, K., \& Xu, H. (2015). The cAMP phosphodiesterase Prune localizes to the mitochondrial matrix and promotes mtDNA replication by stabilizing TFAM. EMBO Reports, 16(4), 520-527. https://doi.org/10.15252/embr.201439636

Zhao, X., León, I. R., Bak, S., Mogensen, M., Wrzesinski, K., Hojlund, K., \& Jensen, O. N. (2010). Phosphoproteome analysis of functional mitochondria isolated from resting human muscle reveals extensive phosphorylation of inner membrane protein complexes and enzymes. Molecular \& Cellular Proteomics, M110, 000299.

Zippin, J. H., Chen, Y., Nahirney, P., Kamenetsky, M., Wuttke, M. S., Fischman, D. A., ... Buck, J. (2003). Compartmentalization of bicarbonate-sensitive adenylyl cyclase in distinct signaling microdomains. The FASEB Journal, 17(1), 82-84. https://doi.org/10.1096/fj.02-0598fje

\section{SUPPORTING INFORMATION}

Additional supporting information may be found online in the Supporting Information section at the end of the article.

Transparent Science Questionnaire for Authors.

How to cite this article: Jakobsen E, Lange SC, Bak LK. Soluble adenylyl cyclase-mediated cAMP signaling and the putative role of PKA and EPAC in cerebral mitochondrial function. J Neuro Res. 2019;97:1018-1038. https://doi. org/10.1002/jnr.24477 